\title{
Verstehensangebote von YouTube-Erklärvideos - Konzeptualisierung und Analyse am Beispiel algebraischer Konzepte
}

\author{
Stefan Korntreff $(\mathbb{D} \cdot$ Susanne Prediger $\mathbb{D}$
}

Eingegangen: 15. Dezember 2020 / Angenommen: 7. September 2021 / Online publiziert: 13. Oktober 2021

(C) Der/die Autor(en) 2021

Zusammenfassung Öffentlich zugängliche Erklärvideos werden immer häufiger genutzt. In Frage gestellt wurde jedoch ihre fachdidaktische Qualität, vor allem inwiefern sie hinreichende Lerngelegenheiten zum Aufbau von konzeptuellem Verständnis für tiefgreifende mathematische Konzepte wie Variable und Term anbieten. Zur theoretisch fundierten und forschungsbasierten Klärung dieser Frage schlägt der Artikel eine fachdidaktische Konzeptualisierung und gegenstandsbezogene Operationalisierung für Verstehensangebote von Erklärvideos vor: als Vorkommen, Auffalten und Vernetzen relevanter Verstehenselemente der Konzepte. Für eine solche Operationalisierung sind die relevanten Verstehenselemente der elementaren Algebra zunächst stoffdidaktisch zu spezifizieren. Die empirische Studie berichtet aus der gegenstandsbezogenen qualitativen Analyse von 50 YouTube-Videos, ausgewählt von den meistgeschauten Kanälen. Die Analyse zeigt, dass alle zu erwartenden Verstehenselemente in den Erklärvideos vorkommen, wenn auch mit unterschiedlichen Priorisierungen (z.B. wenig Unbestimmten-Deutung). Explizit aufgefaltet werden Variablendeutungen im Zusammenhang mit Termen und Gleichungen selten, das Vernetzungspotential ist sehr heterogen. Einige positive Ausnahmen geben Aufschluss darüber, wie Auffalten und Vernetzen umgesetzt und wie hohe

Digitaler Anhang mit den Operationalisierungen der Kodes unter http://www.mathematik.tudortmund.de/ prediger/veroeff/22-JMD-YouTube-Korntreff-Prediger-Erklaervideos-Anhang.pdf

Stefan Korntreff $(\bowtie) \cdot$ Susanne Prediger

Institut für Entwicklung und Erforschung des Mathematikunterrichts, Technische Universität Dortmund, Vogelpothsweg 87, 44227 Dortmund, Deutschland

E-Mail: stefan.korntreff@math.tu-dortmund.de

Susanne Prediger

E-Mail: prediger@math.tu-dortmund.de

Susanne Prediger

IPN Leibniz-Institut für die Pädagogik der Naturwissenschaften und Mathematik, Kiel/Berlin, Deutschland 
Erklärqualität sprachlich realisiert werden kann. Die vorgeschlagene Konzeptualisierung und Operationalisierung der Verstehensangebote von Erklärvideos über die gegenstandsspezifische Analyse der vorkommenden, aufgefalteten und vernetzten Verstehenselemente kann auch für andere fachliche Konzepte adaptiert werden.

Schlüsselwörter Erklärvideo · Verstehensorientierung · Verstehensangebote · Variable $\cdot$ Term $\cdot$ Gleichung

\title{
Conceptual Learning Opportunities of Instructional YouTube Videos - Conceptualization and Analysis for the Case of Algebraic Concepts
}

\begin{abstract}
Publicly available instructional videos are used more and more frequently. However, their subject-specific didactical quality was questioned, especially with respect to the offered learning opportunities to develop conceptual understanding of profound mathematical concepts such as variables and algebraic expressions. For a theoretically sound and research-based clarification of this question, the article suggests a didactical conceptualization and content-specific operationalization for the quality of conceptual learning opportunities in instructional videos, by means of occurrence, unfolding and connecting relevant concept elements. For such an operationalization, the relevant concept elements for algebraic concepts must first be specified epistemologically. The empirical study reports a content-specific qualitative analysis of 50 YouTube videos, selected from the most watched Germanspeaking channels. The analysis shows that all the expected concept elements occur in the videos, albeit with different priorities (e.g. little attention to variables as generalizers). Explicitly unfolded interpretations of variables in connection with expressions and equations are rare, and the potential for connections is very heterogeneous. A few positive exceptions provide insights about how unfolding and connecting can be implemented and by which language high quality explanations can be achieved. The proposed conceptualization and operationalization of quality of the conceptual learning opportunities in instructional videos achieved by contentspecific analysis of the occurrence, unfolding and connecting of concept elements can also be adapted to other subject-related concepts.
\end{abstract}

Keywords Instructional video · Conceptual focus · Conceptual learning opportunities $\cdot$ Variables $\cdot$ Expressions $\cdot$ Equations

\section{Einleitung}

Laut der JIM-Studie 2020 ist YouTube das mit Abstand beliebteste Internetangebot der deutschen Jugendlichen, wobei $21 \%$ der Jugendlichen YouTube-Erklärvideos mehrmals pro Woche auch zu Zwecken formaler Bildung nutzen (MPFS 2020, S. 37, 46). Im Zuge der coronabedingten Schulschließungen griffen mehr als $45 \%$ der von FORSA (2020) befragten 1031 Lehrkräfte (Sekundarstufe I) auf Erklärvideos als Unterrichtsmaterial zurück - die meistgenutzte Ressource nach Aufgabenblättern. 
Angesichts der Nutzungszahlen sind empirische Befunde aus Interventionsstudien zu begrüßen, die belegen, dass mit geeigneten Erklärvideos sowohl prozedurales als auch konzeptuelles Wissen lernwirksam erworben werden kann (Höffler und Leutner 2007; Kant et al. 2017). Hierbei ist der Lernerfolg bzgl. konzeptuellen Wissens allerdings von der fachdidaktischen Angebotsqualität der Videos abhängig (Kulgemeyer 2018). Diese Qualität wurde für online verfügbare Mathematik-Erklärvideos als sehr heterogen beschrieben (Rott 2020) und in Positionspapieren kritisiert (Bersch et al. 2020; Klinger und Walter 2020), z. B. für fehlende Sinnstiftung und fachliche Fehler. Als zentrale fachdidaktische Schwachstelle online-verfügbarer Erklärvideos wurde eine Dominanz kalkülorientierter Erläuterungen von Rechenwegen identifiziert, also die Verstehensorientierung hinterfragt (Bersch et al. 2020; Rott 2020).

Vor diesem Hintergrund stellt sich die Frage, inwiefern anspruchsvolle mathematische Konzepte überhaupt verstehensorientiert in Erklärvideos aufbereitet werden können. Um dieser Frage systematisch nachzugehen, ist eine theoretische und empirische Fundierung der vorliegenden fachdidaktischen Qualitätseinschätzungen notwendig, indem die Verstehensangebote von Erklärvideos theoretisch fundiert konzeptualisiert, gegenstandsbezogen operationalisiert und im Detail analysiert werden.

Der vorliegende Artikel adressiert dieses Forschungsdesiderat exemplarisch für algebraische Konzepte durch eine gegenstandsspezifische Analyse der Verstehensangebote von 50 der meistgeschauten deutschsprachigen YouTube-Erklärvideos zu Variablen, Termen und Gleichungen. Abschn. 2 des Artikels fasst den Hintergrund zu Verstehensangeboten von Erklärvideos und die notwendigen Verstehenselemente in der elementaren Algebra zusammen, Abschn. 3 stellt die herangezogenen Forschungsmethoden vor, während Abschn. 4 Analyseergebnisse berichtet, die in Abschn. 5 diskutiert werden.

\section{Theoretischer Hintergrund}

\subsection{Instruktionspsychologische und fachdidaktische Qualitätskriterien für Erklärvideos}

Aus mediendidaktischer Perspektive sind Erklärvideos ,eigenproduzierte, kurze Filme, in denen Inhalte, Konzepte und Zusammenhänge erklärt werden [...], mit der Intention, beim Betrachter ein Verständnis zu erreichen bzw. einen Lernprozess auszulösen“" (Findeisen et al. 2019, S. 18; ähnlich Wolf 2015). Sie umfassen sowohl Tutorial-Videos mit prozeduralem Fokus, die alltägliche oder mathematische Vorgehensweisen erläutern, als auch Videos mit konzeptuellem Fokus; auf letztere legt dieser Artikel den Schwerpunkt. In der Regel sind Erklärvideos Filme von maximal 20-minütiger Länge, die einen geringen oder mittleren Didaktisierungsgrad vorweisen (Findeisen et al. 2019; Wolf 2015). Manche Videos werden von ausgebildeten Lehrkräften bereitgestellt (bspw. die Kanäle Sebastian Schmidt, Lehrerschmidt, LehrerBros). Entsprechend ihrer prinzipiell didaktischen Intention sind Erklärvideos instruktionale Erklärungen im Videoformat, weshalb sich Qualitätsmerkmale für Erklärvideos aus instruktionspsychologischen Befunden ableiten lassen: Gemäß dem Survey von Wittwer und Renkl (2008) müssen instruktionale Erklärungen (a) auf 
Tab. 1 Beispielkriterien mathematik- und physikdidaktischer Kataloge zur Qualitätserfassung von Erklärvideos. (Marquardt 2016; Bersch et al. 2020; Kulgemeyer und Peters 2016; Kulgemeyer 2018)

\begin{tabular}{lcc}
\hline Inhaltliche Qualität & Kognitive Aktivierung & Adaptivität \\
\hline - Fachliche Korrektheit & - Partiell eigenständige Erarbeitung & - Passung zum \\
- Sprachliche Korrektheit & durch Zuschauende & aktuellen Un- \\
- Einbettung in sinnstiftende Kon- & - Einbindung von Aufgaben zur aktiven & terrichtsstand \\
texte & Auseinandersetzung & - Passung zum \\
- Verstehensorientierung statt reiner & Mediale Gestaltung & individuellen \\
Kalkülorientierung & - Hervorhebung zentraler Inhalte & Vorwissen \\
- Ausreichende Begründung der & - Sinnvolle Segmentierung des Inhalts & \\
Aussagen & & \\
\hline
\end{tabular}

die für ein tieferes Verständnis relevanten Konzepte fokussieren, (b) an die kognitiven Aktivitäten der Lernenden anknüpfen, ohne diese zu ersetzen, und (c) adaptiv sein.

Die in den Fachdidaktiken vorgeschlagenen Qualitätskriterien für Erklärvideos lassen sich den genannten Qualitätsdimensionen der inhaltlichen Qualität (insbesondere die Qualität der Verstehensangebote), der kognitiven Aktivierung und der Adaptivität zuordnen (vgl. Tab. 1). Zur Realisierung kognitiver Aktivierung werden zudem empirisch bestätigte Kriterien medialer Gestaltung betont (etwa Marquardt 2016; Mayer 2009).

In der Mathematikdidaktik wurden Kataloge zur Qualitätserfassung von Erklärvideos bisher vor allem zur praxisnahen Unterstützung von Lehrkräften bei der Videoauswahl formuliert (Marquardt 2016; Bersch et al. 2020), während in der Physikdidaktik Annahmen zur Wirkung von Qualitätskriterien von Physik-Erklärvideos auch empirisch überprüft wurden. So operationalisierten Kulgemeyer und Peters (2016) die Erklärqualität von Online-Physikvideos mit 31 Qualitätskriterien verschiedener Kategorien (u. a. Inhalt, Struktur, Sprache, Mathematisierungsgrad) und zeigten, dass YouTube-spezifische Oberflächenmerkmale (wie View-Anzahl) nicht mit Erklärqualität korrelieren. Kulgemeyer (2018) identifizierte fachdidaktische und instruktionspsychologische Qualitätsmerkmale mit Auswirkung auf die Lernwirksamkeit. Dies waren insbesondere Adaptivität bzgl. Vorwissen und Sprache sowie die Fokussierung auf relevante Inhalte. Letzteres ist der Fokus unseres Artikels.

\subsection{Konzeptualisierung der Verstehensangebote von Erklärvideos}

Wenn in diesem Artikel die Qualität der Verstehensangebote von Erklärvideos anvisiert wird, so wird damit also nur eines der verschiedenen unterrichtspraktisch und empirisch als wichtig identifizierten Kriterien aus Tab. 1 fokussiert. Hiebert und Carpenter (1992, S. 65) beschreiben das Ziel der Verstehensorientierung als ,[one] of the most widely accepted ideas within the mathematics [...]. But achieving this goal has been like searching for the Holy Grail“. Daher lohnt eine genauere Betrachtung der Angebotsseite, auch ohne empirische Verstehensprozesse von Lernenden zu analysieren. Ohne in Lehr-Lernprozessen auch das tatsächlich erreichte Verständnis der Lernenden erfassen zu können, fokussiert das Qualitätskriterium der Verstehensorientierung in erster Linie den inhaltlichen Fokus von Lernangeboten: Werden kalkülhafte Prozeduren oder Konzepte erklärt? Dabei können sich verste- 
hensorientierte Lernangebote durchaus hinsichtlich der Qualität unterscheiden, wie gut die fokussierten Konzepte erklärt werden. Um diese Qualitätsunterschiede zu erfassen, werden nachfolgend die Qualitäten von Verstehensangeboten genauer konzeptualisiert und operationalisiert. Hierfür wird zunächst in Abschn. 2.2.1 theoretisch fundiert, was Verständnis und Prozesse des Verständnisaufbaus ausmacht. In Abschn. 2.2.2 werden daraus Anforderungen an qualitativ gute Verstehensangebote in Erklärungen abgeleitet.

\subsubsection{Theoretischer Hintergrund zu Verständnis und Prozessen des Verständnisaufbaus}

Die Erfassung der Qualität von Lernangeboten wurde in der Unterrichtsqualitätsforschung empirisch systematisiert (vgl. Survey von Hiebert und Grouws 2007). In diesem Artikel wird auf einen Ansatz fokussiert, den Drollinger-Vetter (2011) innerhalb des Pythagoras-Projekts (Reusser und Pauli 2013) entwickelt hat und dieser auf die Analyse von Erklärvideos übertragen. Der Ansatz ist verankert in kognitionswissenschaftlichen Modellen der Wissensorganisation, nach denen Wissen mental in Form strukturierter Wissensnetze repräsentiert wird (Aebli 1994; Hiebert und Carpenter 1992). Konzeptverständnis kann dann über die Vernetztheit relevanter mentaler Repräsentationen definiert werden: Ein Konzept

is understood if its mental representation is part of a network of representations. The degree of understanding is determined by the number and the strength of the connections. [...] [It] is understood thoroughly if it is linked to existing networks with stronger or more numerous connections (Hiebert und Carpenter 1992, S. 67).

Drollinger-Vetter (2011) hat in Anknüpfung an Aebli (1994) vorgeschlagen, Konzeptverständnis mathematikdidaktisch zu charakterisieren als mental repräsentierte Wissensnetze, in denen Konzepte über semantische Verknüpfungen durch Aussagen vernetzt werden. Bspw. werden in den Sätzen ,In Bestimmungsgleichungen gibt es Variablen. Diese Variablen stehen für unbekannte Zahlen.“ die Konzepte Variable, unbekannte Zahl und Bestimmungsgleichung zueinander in Beziehung gesetzt: Verständnis dieser Wissensstruktur umfasst, sowohl die Konzepte als auch deren semantische Beziehungen zueinander ( $x$ steht für $y$ in $z$ ) mental zu konstruieren.

Das Verständnis komplexer mathematischer Konzepte erfordert die mentale Konstruktion unterschiedlicher Grundvorstellungen, d.h. unterschiedlicher normativ bestimmbarer Standard-Deutungen der Konzepte (vom Hofe 1995). Drollinger-Vetter (2011) erfasst dies in ihrem Modell, indem sie die mentalen Repräsentationen mathematischer Konzepte als Zusammensetzung von Verstehenselementen beschreibt. Diese sind definiert als „Teilkonzepte eines Konzepts, die man verstanden haben muss, um das Konzept als Ganzes zu verstehen“ (Drollinger-Vetter 2011, S. 201).

Basierend auf dieser Konzeptualisierung von Verständnis kann Drollinger-Vetter auch Prozesse des Verständnisaufbaus beschreiben: In den Prozessen müssen die relevanten Verstehenselemente mental konstruiert und vernetzt werden. Die Relevanz, Vernetzungen zwischen den Verstehenselementen mental zu konstruieren, ergibt sich aus der Definition von Konzeptverständnis. Sollen die immer komplexer werdenden 
Konzeptstrukturen allerdings mental (weiter-)verarbeitbar bleiben, so bedarf es kognitiver Entlastungsprozesse, die als Verdichten bezeichnet werden:

Der entscheidende Schritt, wie aus Bekanntem Neues entsteht, ist der Schritt des Bildens von Einheiten höherer Ordnung: Dies geschieht durch Verdichten (Objektivieren), das nicht mit Abstraktion verwechselt werden darf. [...] Objektivierungen ermöglichen es, komplexe Strukturen in umfassendere Zusammenhänge einzubauen. Ergebnisse des bisherigen Aufbaus werden in eine Form gebracht, die eine weitere Verarbeitung ermöglicht [...]. (Drollinger-Vetter 2011, S. $64 \mathrm{f}$.)

Erfolgreiche Verdichtungsprozesse sind dadurch gekennzeichnet, dass einerseits im Verdichten zwar Informationen verloren gehen, diese andererseits aber, ,jederzeit durch Auffalten des entsprechenden Elementes in seine dazugehörigen Teilelemente und Beziehungen wieder vollständig hergestellt werden können“ (Drollinger-Vetter 2011, S. 65). Bspw. ließe sich das verdichtete Verstehenselement der Unbekannten auffalten zu der Erläuterung, dass die Variable in Bestimmungsgleichungen für Zahlen steht, die man sucht. Sprachlich zeigen sich Auffaltungsprozesse also durch die ausführliche Explikation der Bestandteile, d.h. durch Erläuterung von Wissenselementen.

Ebenso wie Hiebert und Carpenter (1992) geht Drollinger-Vetter (2011, S. 188 ff.) davon aus, dass sich die Verstehenselemente eines mathematischen Konzepts und deren Verknüpfungen zu bildlichen, sprachlichen und symbolischen Darstellungsformen des Konzepts verdichten lassen. Dementsprechend sind solche Darstellungsformen auch nur dann gehaltvoll interpretierbar, wenn sie zurück zu den relevanten Verstehenselementen und deren Beziehungen zueinander aufgefaltet werden können. Dass diese Auffaltungs-, Vernetzungs- und Verdichtungsprozesse entscheidend für einen inhaltlich gedeuteten Kalkül sind, haben Glade und Prediger (2017) am Beispiel des Brüche-Multiplizierens herausgearbeitet.

\subsubsection{Ableitung von Qualitätsanforderungen an Verstehensangebote (in Erklärungen und Erklärvideos)}

Entsprechend der vorgestellten Beschreibung von Prozessen des Verständnisaufbaus, lassen sich nun drei Anforderungen an Erklärungen ableiten, die darauf zielen, den Verständnisaufbau beim Nutzenden zu unterstützen.

(a) Vorkommen von Verstehenselementen: Zwar ist eine hohe Angebotsqualität von Erklärungen kein Garant für lernendenseitigen Verständnisaufbau (Aebli 1994, S. 99). Trotzdem kann Verständnisaufbau durch Erklärangebote überhaupt nur dann unterstützt werden, wenn in den Erklärungen die relevanten Verstehenselemente des Konzepts bzw. der kalkülhaften Umformungen überhaupt vorkommen. Hiermit wird an den mathematikdidaktischen Konsens hinsichtlich der Verstehensorientierung von Lernangeboten angeknüpft: Erklärungen sollen auf konzeptuelle Gehalte fokussieren und nicht nur auf kalkülhafte Prozeduren (Malle 1993; Prediger 2009). Empirische Evidenzen der Interventions- und Unterrichtsqualitätsforschung, dass das Vorkommen konzeptueller Gehalte in Unterrichtsszenarien lernwirksam für den Aufbau 
von konzeptuellem (und prozeduralem) Verständnis ist, sind im Survey von Hiebert und Grouws (2007) zusammengetragen. Für den deutschsprachigen Raum zeigte Drollinger-Vetter (2011, S. 283), dass das Vorkommen von Verstehenselementen in Unterrichtsstunden zur Einführung des Satzes des Pythagoras in 38 Schulklassen prädiktiv ist für signifikant höhere Lernzuwächse. Somit ist die Qualitätsanforderung des Vorkommens von relevanten Verstehenselementen sowohl theoretisch als auch empirisch von der Interventions- und Unterrichtsqualitätsforschung auf Verstehensangebote in Erklärvideos zu übertragen.

(b) Auffalten von Verstehenselementen: Wittwer und Renkl (2008) haben in ihrem Survey herausgearbeitet, dass lernwirksame instruktionale Erklärungen die fachlich relevanten Konzepte und Prinzipien explizieren müssen, da es andernfalls insbesondere bei leistungsschwächeren Lernenden zu Fehlfokussierungen kommt. Für den lernendenseitigen Verständnisaufbau genügt es also nicht, dass Verstehenselemente in Erklärungen implizit vorkommen, sie müssen auch ausführlich thematisiert werden, d.h. in Anknüpfung an Aebli (1994): Sie müssen aufgefaltet werden, also sprachlich expliziert werden. Da die Unterscheidung von implizit genutzt und explizit aufgefaltet graduell ist, wird sie eine sorgfältige Operationalisierung erfordern (vgl. Methodenteil und digitaler Anhang).

(c) Vernetzen von Verstehenselementen: Insofern Verständnis über die Vernetztheit relevanter mentaler Repräsentationen charakterisiert ist, ist es naheliegend, Verständnisaufbau durch die Explikation dieser Vernetzungen in Erklärungen zu unterstützen: ,there is general consensus that connections between mathematical ideas should be discussed and that students should be encouraged to reflect on them" (Hiebert und Carpenter 1992, S. 86). Entsprechend stellen Wittwer und Renkl (2008) in ihrem Survey heraus, dass lernwirksame instruktionale Erklärungen besonders auch auf die Beziehungen zwischen Konzepten aufmerksam machen müssen.

Die Qualität von Verstehensangeboten von Erklärvideos wird in diesem Artikel also über das Vorkommen, Auffalten und Vernetzen der Verstehenselemente der thematischen Konzepte charakterisiert. Hiermit wird die Verstehensorientierung nicht als Ja/Nein-Kriterium des Vorkommens der Konzepte aufgefasst, sondern einer tiefergehenden fachdidaktischen Exploration zugänglich gemacht. In Anschlussstudien kann die Qualität von Verstehensangeboten von Erklärvideos zu weiteren Qualitätsmerkmalen wie kognitiver Aktivierung und lernendenseitigen Prozessen des Verständnisaufbaus in Beziehung gesetzt werden.

Angesichts der häufig geäußerten Problematisierung der Verstehensorientierung von existierenden Erklärvideos (Bersch et al. 2020; Rott 2020; Klinger und Walter 2020) stellt sich jedoch zunächst die Frage, inwiefern die Verstehensangebote von Erklärvideos für mathematisch tiefliegende Konzepte, etwa die der elementaren Algebra, überhaupt die Qualitätsanforderungen erreichen können. 


\subsection{Stoffdidaktischer Rahmen der gegenstandsbezogenen Operationalisierung von Verstehensangeboten: Verstehens- und Kalkülelemente zentraler algebraischer Konzepte}

Das in Abschn. 2.2 vorgeschlagene Konstrukt der Qualität von Verstehensangeboten muss gegenstandsbezogen operationalisiert werden, indem für den betrachteten Lerngegenstand der elementaren Algebra die relevanten Elemente im Einzelnen spezifiziert werden. Dies erfolgt in diesem Abschnitt unter Rückgriff auf den empirisch fundierten stoffdidaktischen Forschungsstand.

Das wichtigste Konzept der elementaren Algebra ist das Variablenkonzept, weil dessen verschiedene Deutungen auch auf das Term- und Gleichungskonzept unmittelbar Einfluss nehmen (Malle 1993). Die Verstehensangebote von Videos zum Variablenkonzept lassen sich demnach nicht analysieren, ohne die Vernetzung zu diesen Konzepten zu untersuchen (Malle 1993; Drollinger-Vetter 2011, S. 182 f.).

Je nach algebraischer Tätigkeit werden in der mathematikdidaktischen Literatur (unter verschiedenen Namen) mehrere Deutungen der Variable unterschieden und für die Terme und Gleichungen entsprechend abgeleitet (Malle 1993; Freudenthal 1973; Usiskin 1988; Malisani und Spagnolo 2009):

- Variable als Einsetzstelle in der Einsetz-Deutung: Eine zentrale algebraische Tätigkeit in operationaler Perspektive ist das Auswerten von Termen. In dieser Deutung ist die Variable eine Einsetzstelle für Zahlen und ein Term eine Rechenvorschrift, in die Zahlen eingesetzt werden können. Gleichungen sind Aussageformen, die je nach Einsetzung verschiedene Wahrheitswerte haben, z. B. ist $3+x=7$ nur wahr für die Einsetzung $x=4$ (Malle 1993).

- Variable als Unbekannte in erster Zahl-Deutung: In der Zahl-Deutung wird die Variable nicht als Einsetzstelle, sondern als Zahl selbst gedeutet (bei Malle (1993) Gegenstandsaspekt genannt). Die Deutung als unbekannte Zahl ist relevant für die algebraische Tätigkeit des Ermittelns solcher Zahlen aus Bestimmungsgleichungen (Malisani und Spagnolo 2009; Usiskin 1988). Wird die Variable als unbekannte Zahl gedeutet, so ist der Term eine Beschreibung für eine weitere, unbekannte Zahl, bspw. $(x+y) / 2$ das unbekannte arithmetische Mittel von $x$ und $y$, das nun gleichgesetzt werden kann mit einem vorgegebenen Wert.

- Variable als Unbestimmte in zweiter Zahl-Deutung: Für die algebraisch hoch relevante Tätigkeit des Beschreibens allgemeiner Zusammenhänge (z. B. Beziehungen oder Muster, vgl. Prediger und Krägeloh 2016) wird die Variable ebenfalls als Zahl gedeutet, jedoch nicht als unbekannte Zahl, sondern als allgemeine, beliebige Zahl (Kaput 2008; Freudenthal 1973; Usiskin 1988). Terme und Gleichungen mit Variable als Unbestimmter werden in relationaler Sicht nicht als Rechenvorschriften bzw. Aussageformen gedeutet, sondern als Beschreibungsmittel eines allgemeinen Zusammenhangs, z.B. $G \cdot h$ bzw. $V=G \cdot h$, wenn $G$ den Inhalt der Grundfläche und $h$ die Höhe beschreibt.

- Variable als Veränderliche in dritter Zahl-Deutung: Ein Spezialfall der Unbestimmten-Deutung ist die Deutung der Variable als Veränderliche. Sie wird hier als eigene Deutung aufgeführt, um die funktionale Sicht auf Terme und Gleichungen von einer relationalen Sicht zu trennen (bei Malle (1993) Simultan- und Verän- 
derlichenaspekt). Bei der Tätigkeit des Erfassens funktionaler Zusammenhänge wird die Variable als veränderliche Zahl gedeutet. Der Term beschreibt dann eine davon abhängige Zahl, während die Gleichung diesen spezifischen funktionalen Zusammenhang erfasst, etwa $f(x)=3+x$.

- Variable als Symbol ohne Deutung: Eine zentrale algebraische Tätigkeit ist das kalkülhafte Umformen, denn genau daraus bezieht die Algebra ihre große Kraft zur Bewältigung komplexer Zusammenhänge (Hefendehl-Hebeker 2001). Lernende sollten wissen, dass für diese Tätigkeit Variablen als nicht-gedeutete Symbole behandelt werden. Terme und Gleichungen werden dann als syntaktisch wohlgeformte Symbolketten behandelt, ,deren eventuelle Bedeutung man während des Arbeitens vergessen kann" (Malle 1993, S. 47), um sie ausschließlich nach vorgegebenen Regeln umzuformen. Auch diese transformationale Sicht gehört zu einem Verstehen der Algebra (Kieran 2004). Um sie von den inhaltlichen Deutungen abzugrenzen, werden die nicht-gedeuteten Symbole als Kalkülelemente statt als Verstehenselemente bezeichnet.

Sowohl die Unterschiede zwischen operationaler, relationaler und funktionaler Sicht als auch die Unterschiede zwischen der Deutung der Variable als Unbekannte und Unbestimmte wurden in der Algebradidaktik ausführlich diskutiert und empirisch analysiert (Usiskin 1988; Bednarz et al. 1996; Prediger und Krägeloh 2016). Es herrscht inzwischen Konsens, dass Lernende im Laufe der Zeit alle vier Deutungen der Variable und die Konsequenzen für die Deutung von Term und Gleichung lernen müssen (vgl. Übersicht in Tab. 2 als Advance Organizer für weitere Erläuterungen), um die Vielfalt der algebraischen Tätigkeiten ausüben zu können (Bednarz et al. 1996).

Herausfordernd ist hierbei, dass die vier Deutungen der Variable ihre Sinnstiftung aus unterschiedlichen Tätigkeiten beziehen. Bspw. motiviert sich die Variable als Unbekannte über die Tätigkeit, eine unbekannte Zahl aus einer Gleichung zu bestimmen, während die Variable als Unbestimmte aus Generalisierungstätigkeiten ihren Sinn bezieht, zu deren Zweck Zahlwerte absichtlich nicht weiter bestimmt werden, etwa in Rechengesetzen oder Formeln (Freudenthal 1973, S. 294f.).

Auch der Beziehung zwischen den vier inhaltlichen Deutungen und den ungedeuteten Symbolen beim kalkülhaften Umformen (rechte Spalte in Tab. 2) kommt im Aufbau eines Verständnisses für Algebra eine besondere Bedeutung zu (HefendehlHebeker 2001). Diese Vernetzung ist jedoch bei vielen Lernenden nicht adäquat mental konstruiert, obwohl der Erwerb und Transfer gehaltvollen prozeduralen Wissens stets auf Bezüge zu konzeptuellen Bedeutungsgehalten angewiesen ist (Kieran 2013; Malle 1993; Prediger 2009). Demnach muss zum Aufbau dieser Verknüpfung die Bedeutung zweier weiterer Konzepte aufgefaltet werden: Termgleichwertigkeit und Gleichungsäquivalenz, zu denen es neben den Kalkülelementen des regelhaften Umformens (rechte Spalte in Tab. 2) auch Verstehenselemente (in den anderen Spalten) zu lernen gibt:

In der Tätigkeit des kalkülhaften Umformens gelten zwei Terme als gleichwertig, wenn sie mithilfe der Termumformungsregeln ineinander überführt werden können. Um diese kalkülhaften Regeln inhaltlich begründen zu können, muss jedoch die Termgleichwertigkeit auch inhaltlich bestimmt werden (Malle 1993; Prediger 2009): 
Tab. 2 Wissenselemente (Verstehens- und Kalkülelemente) für Konzepte der elementaren Algebra. (Nach Prediger 2020, S. 163; Malle 1993, S. 47)

\begin{tabular}{|c|c|c|c|c|c|}
\hline \multirow[b]{2}{*}{$\begin{array}{l}\text { Tätig- } \\
\text { keiten }\end{array}$} & \multicolumn{4}{|c|}{ Verstehenselemente für verschiedene algebraische Tätigkeiten } & \multirow{2}{*}{$\begin{array}{l}\text { Kalkül- } \\
\text { elemente } \\
\text { Kalkülhaftes } \\
\text { Umformen }\end{array}$} \\
\hline & Auswerten & $\begin{array}{l}\text { Unbekannte } \\
\text { ermitteln }\end{array}$ & $\begin{array}{l}\text { Zusammenhänge } \\
\text { allgemein } \\
\text { erfassen }\end{array}$ & $\begin{array}{l}\text { Veränderungen } \\
\text { erfassen }\end{array}$ & \\
\hline & $\begin{array}{l}\text { Einsetz-Deu- } \\
\text { tung }\end{array}$ & $\begin{array}{l}\text { Zahl-Deutung } \\
\text { als Unbekann- } \\
\text { te }\end{array}$ & $\begin{array}{l}\text { Zahl-Deutung } \\
\text { als Unbe- } \\
\text { stimmte }\end{array}$ & $\begin{array}{l}\text { Zahl-Deutung } \\
\text { als Veränderli- } \\
\text { che }\end{array}$ & $\begin{array}{l}\text { Symbol ohne } \\
\text { Deutung }\end{array}$ \\
\hline Variable & $\begin{array}{l}\text { Einsetzstelle } \\
\text { für Zahlen }\end{array}$ & $\begin{array}{l}\text { Steht für un- } \\
\text { bekannte Zahl, } \\
\text { die man sucht }\end{array}$ & $\begin{array}{l}\text { Steht für alle } \\
\text { Zahlen, für die } \\
\text { man verallge- } \\
\text { meinert }\end{array}$ & $\begin{array}{l}\text { Steht für eine } \\
\text { Größe, die } \\
\text { sich verändert }\end{array}$ & $\begin{array}{l}\text { Nicht gedeute- } \\
\text { tes Symbol }\end{array}$ \\
\hline Term & $\begin{array}{l}\text { Rechenvorschrift, } \\
\text { in die Zahlen } \\
\text { eingesetzt } \\
\text { werden } \\
\text { können } \\
\text { (operationale } \\
\text { Sicht) }\end{array}$ & $\begin{array}{l}\text { Beschreibung } \\
\text { für eine weite- } \\
\text { re unbekannte } \\
\text { Zahl }\end{array}$ & $\begin{array}{l}\text { Allgemeine } \\
\text { Beschreibung } \\
\text { eines Zusam- } \\
\text { menhangs } \\
\text { (relationale } \\
\text { Sicht) }\end{array}$ & $\begin{array}{l}\text { Allgemeine } \\
\text { Beschreibung } \\
\text { eines funk- } \\
\text { tionalen } \mathrm{Zu}- \\
\text { sammenhangs } \\
\text { (funktionale } \\
\text { Sicht) }\end{array}$ & $\begin{array}{l}\text { Nicht gedeu- } \\
\text { tete Symbol- } \\
\text { Kette (nach } \\
\text { Konstruktions- } \\
\text { regeln) }\end{array}$ \\
\hline $\begin{array}{l}\text { Term- } \\
\text { gleich- } \\
\text { wertig- } \\
\text { keit }\end{array}$ & $\begin{array}{l}\text { Einsetzungs- } \\
\text { gleichheit: } \\
2 \text { Terme er- } \\
\text { geben für alle } \\
\text { Einsetzungen } \\
\text { denselben } \\
\text { Wert }\end{array}$ & - & $\begin{array}{l}\text { Beschrei- } \\
\text { bungsgleich- } \\
\text { heit: } 2 \text { Terme } \\
\text { beschreiben } \\
\text { dasselbe (Bild, } \\
\text { Situation, } \\
\text { Zusammen- } \\
\text { hang ...) }\end{array}$ & $\begin{array}{l}\text { Beschrei- } \\
\text { bungsgleich- } \\
\text { heit: } 2 \text { Terme } \\
\text { beschreiben } \\
\text { denselben } \\
\text { funktionalen } \\
\text { Zusammen- } \\
\text { hang }\end{array}$ & $\begin{array}{l}\text { Umformungs- } \\
\text { gleichheit: } \\
\text { 1. Term in } \\
\text { 2. Term nach } \\
\text { festen Umfor- } \\
\text { mungsregeln } \\
\text { überführbar }\end{array}$ \\
\hline $\begin{array}{l}\text { Glei- } \\
\text { chung }\end{array}$ & $\begin{array}{l}\text { Aussageform, } \\
\text { in der durch } \\
\text { Einsetzen } \\
\text { potenzielle } \\
\text { Lösungen ge- } \\
\text { prüft werden } \\
\text { können }\end{array}$ & $\begin{array}{l}\text { Bestimmungs- } \\
\text { gleichung als } \\
\text { Bedingung, } \\
\text { aus der unbe- } \\
\text { kannte Zahl } \\
\text { herauszufinden } \\
\text { ist }\end{array}$ & $\begin{array}{l}\text { Allgemein- } \\
\text { gültige Bedin- } \\
\text { gung für alle } \\
\text { Zahlen }\end{array}$ & $\begin{array}{l}\text { Allgemeine } \\
\text { Beschrei- } \\
\text { bung eines } \\
\text { (funktionalen) } \\
\text { Zusammen- } \\
\text { hangs }\end{array}$ & $\begin{array}{l}\text { Nicht gedeu- } \\
\text { tete Symbol- } \\
\text { Kette }\end{array}$ \\
\hline $\begin{array}{l}\text { Glei- } \\
\text { chungs- } \\
\text { äquiva- } \\
\text { lenz }\end{array}$ & $\begin{array}{l}2 \text { Gleichungen } \\
\text { haben dieselbe } \\
\text { Lösungsmenge } \\
\text { (Wahrmacher) }\end{array}$ & $\begin{array}{l}2 \text { Gleichungen } \\
\text { beschreiben } \\
\text { dieselben } \\
\text { unbekannten } \\
\text { Zahlen }\end{array}$ & - & - & $\begin{array}{l}\text { 1. Gleichung } \\
\text { in } 2 \text {. Glei- } \\
\text { chung nach } \\
\text { festen Äqui- } \\
\text { valenzumfor- } \\
\text { mungsregeln } \\
\text { überführbar }\end{array}$ \\
\hline
\end{tabular}

- Wird die Variable als Einsetzstelle gedeutet, so lässt sich die Gleichwertigkeit von Termen als Einsetzungsgleichheit interpretieren: Zwei Terme sind dann gleichwertig, wenn sie für alle eingesetzten Zahlen denselben Wert ergeben.

- Wird die Variable dagegen als Unbestimmte oder Veränderliche gedeutet, dann lässt sich die Gleichwertigkeit von Termen als Beschreibungsgleichheit interpretieren: Zwei Terme sind dann gleichwertig, wenn sie denselben (ggf. funktionalen) Zusammenhang beschreiben. 
Analog lassen sich Äquivalenzumformungen von Gleichungen inhaltlich begründen, indem das Konzept Gleichungsäquivalenz in Einsetz- oder Unbekannten-Deutung gedeutet wird (Tab. 2).

Zum Verständnis von Termen und Gleichungen gehört also auch, deren kalkülhafte Umformungsregeln mit entsprechenden inhaltlichen Deutungen der Konzepte Termgleichwertigkeit und Gleichungsäquivalenz zu vernetzen (Malle 1993; Prediger 2009). Dies geht über eine reine Darstellungsvernetzung von bildlicher, kontextueller und symbolischer Darstellung von Term und Gleichung hinaus, weil die Anforderungen an Termgleichwertigkeit bzw. Gleichungsäquivalenz inhaltlich verstanden werden müssen, damit die Regeln nicht beliebig sind (Hefendehl-Hebeker 2001). Somit erfordern auch die Gleichwertigkeitskonzepte, die den Umformungsregeln zugrunde liegen, eine inhaltliche Deutung (Kieran 2013).

Für eine begrifflich scharfe Fassung der Beziehungen zwischen Inhalt und Kalkül werden in diesem Artikel also zusammenfassend folgende Bezeichnungen genutzt (vgl. Tab. 2):

- die verschiedenen Grundvorstellungen der einzelnen Konzepte (in den Zellen) werden als Verstehenselemente bezeichnet,

- die Verstehenselemente werden spaltenweise zu Deutungen zusammengefasst, indem die Vorstellungen von Term, Gleichung etc. den verschiedenen VariablenDeutungen zugeordnet werden (bspw. Term in Einsetz-Deutung entspricht der operationalen Termvorstellung),

- die symbolisch-kalkülhafte Vorstellungen der Konzepte (rechte Spalte) werden (in den Zellen) Kalkülelemente genannt,

- Verstehenselemente und Kalkülelemente werden unter dem Oberbegriff Wissenselemente erfasst.

Die Analyse der Verstehensangebote der Erklärvideos wird somit über das Vorkommen, Auffalten und Vernetzen der in Tab. 2 aufgeführten Wissenselemente operationalisiert.

Bei der Analyse von Erklärvideos stößt man unweigerlich auch auf typische Beispiele für didaktisch nicht tragfähige „Deutungen“ kalkülhafter Umformungen: etwa die „Obstsalat-Algebra“ (Arcavi et al. 2017) mit ihrer Fehlvorstellung des Buchstaben als Gegenstand (Küchemann 1981), in der Variablen nicht als Anzahlen, sondern als (physische) Gegenstände wie Obstsorten gedeutet werden. Diese oft für die Begründung der Termumformungsregel ,Zusammenfassen“ herangezogene Fehlvorstellung wurde empirisch identifiziert als verstehenshinderlich und problematisch langlebig (Arcavi et al. 2017). MacGregor und Stacey (1997) betonen zudem, dass Lernende sie selten selbstständig entwickeln, sondern oft durch nicht tragfähige Instruktion erwerben. Die Nutzung der Obstsalat-Deutung kann also als Beispiel für eine gutgemeinte, aber unpassend realisierte Orientierung am Verstehen interpretiert werden, worauf in den nachfolgenden Analysen zurückzukommen sein wird.

\subsection{Forschungsfragen}

Zusammenfassend lässt sich festhalten: Ausgehend von Drollinger-Vetters (2011) Verstehensmodell wurden Qualitätsanforderungen an Verstehensangebote von Er- 
klärvideos zur elementaren Algebra konzeptualisiert als Vorkommen, Auffalten und Vernetzen der in Tab. 2 aufgeführten Wissenselemente (Verstehens- und Kalkülelemente) für zentrale algebraische Konzepte. Für das Auffalten sind ausführliche Explikationen der Konzepte und ihrer Wissenselemente bedeutsam, für das Vernetzen zusätzlich die sprachlich explizite Erläuterung der Beziehung zwischen den aufgefalteten Wissenselementen. Aus algebradidaktischer Sicht sind sowohl das Vernetzen der Deutungen als auch das Vernetzen verschiedener Konzepte relevant. Bezüglich der Kalkülelemente ist vor allem deren Vernetzung mit den Verstehenselementen inhaltlich bedeutsam. Aus dieser Operationalisierung ergeben sich zwei Forschungsfragen:

(FF1) Welche Verstehenselemente zu den Konzepten Variable, Term und Gleichung kommen (a) in den YouTube-Erklärvideos vor, werden (b) aufgefaltet und (c) miteinander vernetzt?

(FF2) Welche Kalkülelemente zu Variable, Term, Termgleichwertigkeit, Gleichung und Gleichungsäquivalenz kommen (a) in den YouTube-Erklärvideos vor, werden (b) aufgefaltet und (c) mit relevanten Verstehenselementen vernetzt?

\section{Methodisches Vorgehen}

\subsection{Methoden der Datenerhebung}

Das Datenkorpus für die Analyse der Verstehensangebote wurde zusammengestellt aus 50 Erklärvideos von 28 der meistgenutzten deutschsprachigen YouTube-Kanäle für Mathematik-Erklärvideos. Die 50 Erklärvideos sind zwischen 2:37 und 20:06 min lang (im arithmetischen Mittel 6:34 min). Die Kanalauswahl (Tab. 3) erfolgte über die Kanalreichweite, operationalisiert durch Anzahl der Kanalabos (am 07.09.2020). Dieses Samplingkriterium wurde gewählt, da der YouTube-Algorithmus den Zuschauenden personalisiert „,beliebte“ Videos vorschlägt (viele Views, Likes, Kommentare, etc.), daher ist die fachdidaktische Qualität beliebter Videos besonders entscheidend; beliebte Kanäle statt beliebte Videos ermöglichen zudem eine gewisse Breite der Anbieter.

Zusätzlich wurden zwei Kanäle mit 5 Videos einbezogen, von denen eine hohe Qualität zu erwarten ist: Sebastian Schmidt als Gewinner des Deutschen Lehrerpreises 2019 für sein Konzept mit Erklärvideo-Einsatz und KhanAcademyDeutsch als deutschsprachiges YouTube-Angebot der einflussreichen US-amerikanischen videobasierten Lernplattform, deren Lernwirksamkeit empirisch nachgewiesen wurde (Weeraratne und Chin 2018).

Zentrales Sampling-Kriterium für die Auswahl der einzelnen Videos aus den Kanälen war der thematische Fokus auf die fünf Konzepte Variable, Term, Gleichung sowie Termgleichwertigkeit und Gleichungsäquivalenz. Ausgeschlossen wurden u. a. Videos zu binomischen Formeln, linearen Gleichungssystemen und Funktionen, um für die fünf fokussierten Konzepte ein thematisch homogeneres Datenkorpus zu 
Tab. 3 Datenkorpus der 50 Erklärvideos mit Quellen und fokussierten algebraischen Konzepten

\begin{tabular}{|c|c|c|c|c|c|c|c|c|}
\hline \multicolumn{2}{|c|}{ Sample: 28 Kanäle } & \multicolumn{2}{|c|}{50 Videos } & \multicolumn{5}{|c|}{ Fokussierte Konzepte } \\
\hline Kanal & $\begin{array}{l}\text { Abos } \\
(07.09 .2020)\end{array}$ & Nr. & $\begin{array}{l}\text { Eindeutige YouTube- } \\
\text { Kennung } \\
\text { (Link youtube.com/ } \\
\text { watch?v=...) }\end{array}$ & $\begin{array}{l}V \\
13 x\end{array}$ & $\begin{array}{l}\mathrm{T} \\
18 \mathrm{x}\end{array}$ & $\begin{array}{l}\text { TG } \\
20 x\end{array}$ & $\begin{array}{l}\mathrm{G} \\
8 \mathrm{x}\end{array}$ & $\begin{array}{l}\mathrm{GÄ} \\
18 \mathrm{x}\end{array}$ \\
\hline \multirow{3}{*}{$\begin{array}{l}\text { Mathe - } \\
\text { simpleclub }\end{array}$} & 796.000 & V1 & $\mathrm{v}=\mathrm{sGjB} 6 \mathrm{frA} 1 \mathrm{LI}$ & + & - & - & - & - \\
\hline & & $\mathrm{V} 2$ & $\mathrm{v}=\mathrm{EkfV} 1 \mathrm{GkZ224}$ & - & + & - & + & - \\
\hline & & V3 & $\mathrm{v}=\mathrm{K} 8 \mathrm{CNFqlxeM0}$ & - & - & - & - & + \\
\hline \multirow{5}{*}{$\begin{array}{l}\text { Mathe by } \\
\text { Daniel Jung }\end{array}$} & 681.000 & V4 & $v=Y w-u 7 P 39 C h Q$ & - & + & - & + & - \\
\hline & & V5 & $\mathrm{v}=\mathrm{gbkHViLUYfM}$ & - & + & - & - & - \\
\hline & & V6 & $\mathrm{v}=1 \mathrm{LZei} 3 \mathrm{FUjMg}$ & - & - & - & + & + \\
\hline & & V7 & $\mathrm{v}=9 \mathrm{UVkZFySE5I}$ & - & - & - & - & + \\
\hline & & V8 & $\mathrm{v}=\mathrm{RWXxwPAwS0}$ & - & - & - & + & + \\
\hline \multirow[t]{3}{*}{ Lehrerschmidt } & 571.000 & V9 & $\mathrm{v}=\mathrm{pOMQNP5S8IE}$ & - & + & + & - & - \\
\hline & & V10 & $v=\_v N 7 N X v Q b K E$ & - & - & + & - & + \\
\hline & & V11 & $\mathrm{v}=\mathrm{G} 5 \mathrm{XxS} 2 \mathrm{OFsJU}$ & - & - & - & - & + \\
\hline \multirow[t]{3}{*}{ Schoolseasy } & 131.000 & V12 & v=hvd_kUY0ETM & + & - & - & - & - \\
\hline & & V13 & $\mathrm{v}=\mathrm{Xq} 9 \mathrm{oR} 45 \mathrm{G} 2 \times 4$ & - & + & + & - & - \\
\hline & & V14 & $\mathrm{v}=\mathrm{RwSBJROJe6Q}$ & - & - & - & + & + \\
\hline Lehrer MaPhy & 72.000 & V15 & $\mathrm{v}=\mathrm{pYzi} 70 \mathrm{ooJGE}$ & - & - & - & - & + \\
\hline Herr Mathe & 52.500 & V16 & $\mathrm{v}=\mathrm{CVnU}-\mathrm{qdYXgU}$ & - & - & + & - & + \\
\hline \multirow{3}{*}{$\begin{array}{l}\text { Musstewissen } \\
\text { Mathe }\end{array}$} & 52.400 & V17 & $\mathrm{v}=\mathrm{ox} 2 \mathrm{BtxWyccY}$ & + & - & + & - & - \\
\hline & & V18 & $\mathrm{v}=3 \mathrm{RW} \operatorname{grC} 6 \mathrm{kNSU}$ & - & + & + & - & - \\
\hline & & V19 & $\mathrm{v}=0$ ioTiR0HLV0 & - & - & - & + & + \\
\hline Matheretter & 49.800 & V20 & $\mathrm{v}=\mathrm{OF} 2 \mathrm{AZX}$ 9D108 & + & + & - & - & + \\
\hline StrandMathe & 32.100 & V21 & $\mathrm{v}=$ VIEyxlxD7Dk & + & + & - & - & - \\
\hline $\begin{array}{l}\text { Koonys Schu- } \\
\text { le }\end{array}$ & 31.000 & V22 & $\mathrm{v}=\mathrm{PWr} 2 \mathrm{nTCK} \_\mathrm{LA}$ & - & - & - & - & + \\
\hline \multirow{5}{*}{$\begin{array}{l}\text { KhanAca- } \\
\text { demyDeutsch }\end{array}$} & 20.000 & V23 & $\mathrm{v}=8 \mathrm{oFdV} 6 \mathrm{~F} 64 \mathrm{tw}$ & + & - & - & - & - \\
\hline & & V24 & $\mathrm{v}=\mathrm{JS} Y 9 Z \mathrm{Zd} 8 \mathrm{vGMY}$ & - & - & + & - & - \\
\hline & & V25 & $\mathrm{v}=\mathrm{Tk}-\mathrm{RZAbcb} 48$ & - & - & + & - & - \\
\hline & & V26 & $\mathrm{v}=$ NomcIjSKqfY & - & - & - & - & + \\
\hline & & V27 & $\mathrm{v}=$ DztIUBit0SE & - & - & - & + & + \\
\hline $\begin{array}{l}\text { Mathematik } \\
\text { Nachhilfe }\end{array}$ & 17.900 & V28 & $\mathrm{v}=\mathrm{uh} 4 \mathrm{sr} Y \mathrm{JkVe} 8$ & - & + & + & - & - \\
\hline $\begin{array}{l}\text { Mathias Pie- } \\
\text { per }\end{array}$ & 14.400 & V29 & $\mathrm{v}=1 \mathrm{AFGkbC8fTk}$ & + & + & - & + & - \\
\hline Kapiert.de & 6550 & V30 & $\mathrm{v}=\mathrm{VUSSe} 2 \mathrm{YPYzI}$ & + & - & - & - & - \\
\hline $\begin{array}{l}\text { Nachhilfe- } \\
\text { channel }\end{array}$ & 6120 & V31 & $\mathrm{v}=\mathrm{Gmwsts} 0 \mathrm{HtkA}$ & - & - & + & - & - \\
\hline \multirow{5}{*}{$\begin{array}{l}\text { Sebastian } \\
\text { Schmidt }\end{array}$} & 5940 & V32 & $\mathrm{v}=\mathrm{IW} 5 \mathrm{rFKue} 1 \mathrm{hw}$ & - & + & - & - & - \\
\hline & & V33 & v=jMB4YiJq2qg & - & + & - & - & - \\
\hline & & V34 & $v=b \_f O z k X 5 \times h o$ & - & - & + & - & - \\
\hline & & V35 & $\mathrm{v}=\mathrm{BIlOPtIuZ9U}$ & - & - & + & - & - \\
\hline & & V36 & v=Dz1UIISQuQg & - & - & - & - & + \\
\hline
\end{tabular}


Tab. 3 (Fortsetzung)

\begin{tabular}{|c|c|c|c|c|c|c|c|c|}
\hline \multicolumn{2}{|c|}{ Sample: 28 Kanäle } & \multicolumn{2}{|c|}{50 Videos } & \multicolumn{5}{|c|}{ Fokussierte Konzepte } \\
\hline Kanal & $\begin{array}{l}\text { Abos } \\
(07.09 .2020)\end{array}$ & Nr. & $\begin{array}{l}\text { Eindeutige YouTube- } \\
\text { Kennung } \\
\text { (Link youtube.com/ } \\
\text { watch?v=...) }\end{array}$ & $\begin{array}{l}\mathrm{V} \\
13 \mathrm{x}\end{array}$ & $\begin{array}{l}\mathrm{T} \\
18 \mathrm{x}\end{array}$ & $\begin{array}{l}\text { TG } \\
20 x\end{array}$ & $\begin{array}{l}G \\
8 x\end{array}$ & $\begin{array}{l}\text { Gë } \\
18 \mathrm{x}\end{array}$ \\
\hline $\begin{array}{l}\text { Young- } \\
\text { Business- } \\
\text { School }\end{array}$ & 5680 & V37 & $\mathrm{v}=\mathrm{VpeCrV} 2 \mathrm{dS} 4 \mathrm{~s}$ & + & + & - & - & - \\
\hline LehrerBros & 4760 & V38 & $\mathrm{v}=\mathrm{auFMYVZDErw}$ & - & + & + & - & - \\
\hline 52 Volt & 4060 & V39 & $\mathrm{v}=\mathrm{cNCNsG \_ mjnE}$ & - & - & - & - & + \\
\hline Classninjas & 3690 & V40 & $\mathrm{v}=\mathrm{cTyFYHhV81A}$ & + & + & - & - & - \\
\hline Herr Mauch & 3580 & V41 & v=ap1ZTaIk-rE & + & + & - & - & - \\
\hline ObachtMathe & 2290 & V42 & $\mathrm{v}=$ GrIY23QctJM & - & - & + & - & - \\
\hline Videolernen & 1380 & V43 & $\mathrm{v}=\mathrm{rJXF} 4 \mathrm{XNI} 2 \mathrm{Y} 0$ & - & - & + & - & - \\
\hline $\begin{array}{l}\text { Mathe-lern- } \\
\text { tipps.de }\end{array}$ & 529 & V44 & $\mathrm{v}=\mathrm{FQbFAPKMmDk}$ & + & - & + & - & - \\
\hline $\begin{array}{l}\text { Vorneauf- } \\
\text { derwelle }\end{array}$ & 148 & V45 & $\mathrm{v}=0 \mathrm{mYrd} 9 \mathrm{MDgU} 0$ & + & + & - & - & - \\
\hline \multicolumn{9}{|c|}{ Kalkülvideos (Artikulierte Intention: Wiederholung von Kalkül) } \\
\hline Herr Mathe & 52.500 & V46 & $\mathrm{v}=\mathrm{ge} 2 \mathrm{cQgr} 9 \mathrm{~s} 4 \mathrm{~g}$ & - & - & + & - & - \\
\hline StrandMathe & 32.100 & V47 & $\mathrm{v}=2 \mathrm{gxaRrS5YD} 4$ & - & - & - & - & + \\
\hline Mathehilfe24 & 31.400 & V48 & $v=$ Py6BnvchTKA & - & - & + & - & - \\
\hline $\begin{array}{l}\text { Mathema- } \\
\text { Trick }\end{array}$ & 11.000 & V49 & $\mathrm{v}=7 \mathrm{LBV} 0913 \mathrm{sLA}$ & - & - & + & - & - \\
\hline Caaosstv & 1400 & V50 & $\mathrm{v}=\mathrm{zDctDgGKF} 58$ & - & + & + & - & + \\
\hline
\end{tabular}

$V$ Variable, $T$ Term, $T G$ Termgleichwertigkeit, $G$ Gleichung, $G \ddot{A}$ Gleichungsäquivalenz

erhalten. In Fällen, in denen Kanäle mehrere Videos zu den untersuchten Konzepten anboten (bspw. Mathe by Daniel Jung), wurden die Videos zufällig ausgewählt.

19 auf Umformungen fokussierende Videos wurden hinzugenommen, um die Vernetzung zu den Verstehenselementen zu untersuchen (FF2c). Von diesen 19 Videos sind nur 5 allein auf das Wiederholen des Umform-Kalküls fokussiert. Die übrigen 14 Videos können auch darauf untersucht werden, inwiefern Rückgriffe auf Verstehenselemente zur Termgleichwertigkeit bzw. Gleichungsäquivalenz vorkommen. Denn 9 der 14 Videos artikulieren in ihrem Videotitel oder im Video selbst die Intention, Umformungsverfahren neu einzuführen (,Wir beschäftigen uns gleich mal zu Anfang [der 7. Klasse] mit dem wichtigen Thema Gleichungen lösen." V15, 0:01), und die übrigen 5 wollen bei Verständnisproblemen bzgl. Umformungen unterstützen (,,[D]iese Gleichungen soll man dann umstellen und das finden ganz schön viele Leute ziemlich verwirrend." V22, 0:07). Die Kontrastierung mit den 5 reinen Kalkülvideos (V46-V50 in Tab. 3) dient der Validierung des Kategoriensystems, denn eine Analyse der Verstehensangebote sollte es ermöglichen, kalkülorientierte Erklärungen ohne vorkommende Verstehenselemente zu identifizieren. 


\subsection{Methoden der Datenanalyse und -auswertung}

Die 50 Erklärvideos wurden inklusive der relevanten Bildelemente (Screenshots) und Zeigegesten (kursiv in eckigen Klammern) transkribiert und dann in fünf Schritten qualitativ analysiert:

1. Von Erklärvideos mit Fokus auf das Variablenkonzept ist nicht in gleichem Maße Vorkommen, Auffalten und Vernetzen von Verstehenselementen des Gleichungskonzepts zu erwarten, wie von Videos mit explizitem Fokus auf das Gleichungskonzept. Deshalb wurden für jedes Konzept diejenigen Fokusvideos identifiziert, in denen das Konzept fokussiert wird (unabhängig vom Vorkommen im Titel). Die Kodierung nach fokussierten Konzepten und die Anzahl der Fokusvideos zu jedem Konzept sind in Tab. 3 aufgeführt.

2. Die deduktive Kodierung (Mayring 2015) erfolgte in kleinen Sinnabschnitten, um trotz hochinferentem Charakter Reliabilität zu erreichen. Dazu wurden die themenbezogenen Phasen der Videos (Abschnitte ohne Werbung, Ankündigungen etc.) in Sinnabschnitte von zirka 30 s unterteilt. Für jeden Sinnabschnitt wurden die vorkommenden Wissenselemente (FF1a und FF2a) mit den Kategorien aus Tab. 4 kodiert, unter Berücksichtigung der Bildelemente und Zeigegesten. Die Operationalisierung der Kodes ergibt sich durch die Erläuterung in Tab. 2. Das Vorkommen eines Wissenselements wird kodiert, wenn es nur genutzt wird oder sein Verständnis zur Erfassung eines anderen Wissenselements vorausgesetzt wird. Ein Auszug aus dem Kodiermanual findet sich im digitalen Anhang des Artikels. Die Deutungen der Veränderlichen kamen (durch Ausschließen von Videos zu Funktionen) insgesamt nur in 5 von 639 kodierten Abschnitten vor. Diese Stellen wurden daher unter Unbestimmte subsumiert (vgl. Tab. 4). Die Fehlvorstellung des Buchstaben als Gegenstand kam in den Videos immer wieder vor und wurde deshalb als unpassende „Deutung“ unter dem Kode BuchstabeAlsGegenstand miterfasst. Ein Beispiel hierzu findet sich in Abschn. 4.2.1.

3. Zur Erfassung des Auffaltens der Wissenselemente (FF1b und FF2b) wurde für jedes der in Schritt 2 identifizierten Wissenselemente entschieden, ob es expli-

Tab. 4 Kodes für die vorkommenden Wissenselemente

\begin{tabular}{|c|c|c|c|c|}
\hline \multirow[b]{2}{*}{ Konzept } & \multicolumn{3}{|c|}{ Verstehenselemente des Konzepts } & \multirow{2}{*}{$\begin{array}{l}\text { Kalkülelemente } \\
\text { Symbol ohne } \\
\text { Deutung }\end{array}$} \\
\hline & Einsetz-Deutung & $\begin{array}{l}\text { Unbekannten- } \\
\text { Deutung }\end{array}$ & $\begin{array}{l}\text { Unbestimmten- } \\
\text { Deutung }\end{array}$ & \\
\hline Variable & V-Einsetzstelle & V-Unbekannte & V-Unbestimmte & V-Symbol \\
\hline Term & $\begin{array}{l}\text { T-Rechenvor- } \\
\text { schrift }\end{array}$ & T-Zahl & $\begin{array}{l}\text { T-Beschreibungs- } \\
\text { mittel }\end{array}$ & T-Symbolkette \\
\hline $\begin{array}{l}\text { Termgleich- } \\
\text { wertigkeit }\end{array}$ & $\begin{array}{l}\text { TG-Einsetzungs- } \\
\text { gleichheit }\end{array}$ & - & $\begin{array}{l}\text { TG-Beschreibungs- } \\
\text { gleichheit }\end{array}$ & $\begin{array}{l}\text { TG-Umfor- } \\
\text { mung }\end{array}$ \\
\hline Gleichung & G-Aussageform & $\begin{array}{l}\text { G-Bestimmungs- } \\
\text { gleichung }\end{array}$ & $\begin{array}{l}\text { G-allgemeine } \\
\text { Bedingung }\end{array}$ & G-Symbolkette \\
\hline $\begin{array}{l}\text { Gleichungs- } \\
\text { äquivalenz }\end{array}$ & $\begin{array}{l}\text { GÄ-gleiche } \\
\text { Lösungsmenge }\end{array}$ & $\begin{array}{l}\text { GÄ-gleiche } \\
\text { Unbekannte }\end{array}$ & - & $\begin{array}{l}\text { GÄ-Umfor- } \\
\text { mung }\end{array}$ \\
\hline
\end{tabular}

Veränderliche wurde wegen geringem Vorkommen unter Unbestimmten-Deutung subsumiert 
zit erklärt wird, also aufgefaltet, oder ob es nur ohne weitere Explikation genutzt wird (vgl. Beispiele im digitalen Anhang). Das explizite Auffalten wurde mit Suffix -auff am Kode gekennzeichnet. Rein kalkülorientierte Videos lassen sich dann dadurch charakterisieren, dass sie nur Kalkülelemente auffalten und keine Deutungen.

4. Für das Vernetzen der Deutungen für ein Konzept (FF1c) wurden alle Videos identifiziert, die mehr als ein Verstehenselement eines Konzepts auffalten. Für die je zwei aufgefalteten Verstehenselemente wurde erfasst, ob eine Vernetzung zwischen den beiden Verstehenselementen im Video sprachlich expliziert wird. Analog wurde für das Vernetzen von Konzepten (FF1c) kodiert, ob aufgefaltete Verstehenselemente unterschiedlicher Konzepte sprachlich explizit zusammengeführt wurden. Für das Vernetzen der Kalkülelemente mit den Verstehenselementen wurde die Explikation der Vernetzung zwischen aufgefalteten Kalkül- und Verstehenselementen eines Konzepts beurteilt (FF2c). Auszüge aus dem Kodiermanual finden sich im digitalen Anhang. Insgesamt wurde über 138 mögliche Vernetzungen entschieden.

5. Schließlich wurde die Interraterreliabilität bestimmt. Dazu wurden die fokussierten Konzepte, die Fehlvorstellung des Buchstaben als Gegenstand und die Vernetzung aller Videos von einer zweiten unabhängigen Raterin kodiert, für das Vorkommen und Auffalten der Verstehenselemente $20 \%$ des Datenmaterials. Ja/ Nein-Entscheidungen werden mittels des Übereinstimmungsmaßes Yules Y berichtet (Wirtz und Caspar 2002). Für die Kodierung der in den Videos fokussierten Konzepte ergab sich mindestens $\mathrm{Y}=0,79$; für die Kodierung des Vorkommens der Verstehenselemente mindestens $\mathrm{Y}=0,87$; für das Auffalten $\mathrm{Y}=0,81$ und für die Vernetzung Y=0,64. Bzgl. des Vorkommens der Fehlvorstellung herrschte $100 \%$ Übereinstimmung. Die Übereinstimmung bzgl. der Vernetzung können damit als gut, die übrigen als sehr gut eingeschätzt werden (Wirtz und Caspar 2002). Nichtübereinstimmungen wurden durch Konsensurteil bereinigt.

\section{Empirische Analyse}

\subsection{Vorkommen, Auffalten und Vernetzen von Deutungen der Konzepte Variable, Term und Gleichung}

Forschungsfrage FF1 erfasst die Verstehensangebote eines Erklärvideos zu den Konzepten Variable, Term und Gleichung durch das Vorkommen, Auffalten und Vernetzen der relevanten Verstehenselemente. Die Vernetzung zu den Kalkülelementen wird in Abschn. 4.2 untersucht. Konkretisierungen erfolgen in beiden Abschnitten an wenigen Beispiel-Videos, an denen sich in Passagen überschaubarer Länge viele für die Analyse relevante Beobachtungen nachvollziehen lassen.

\subsubsection{Vorkommen und Auffalten der Deutungen der Konzepte}

Gelegenheiten zum Konzeptverstehen bieten Erklärungen nur, wenn die notwendigen Verstehenselemente vorkommen (Abschn. 2.2). Das Transkript aus dem Video 


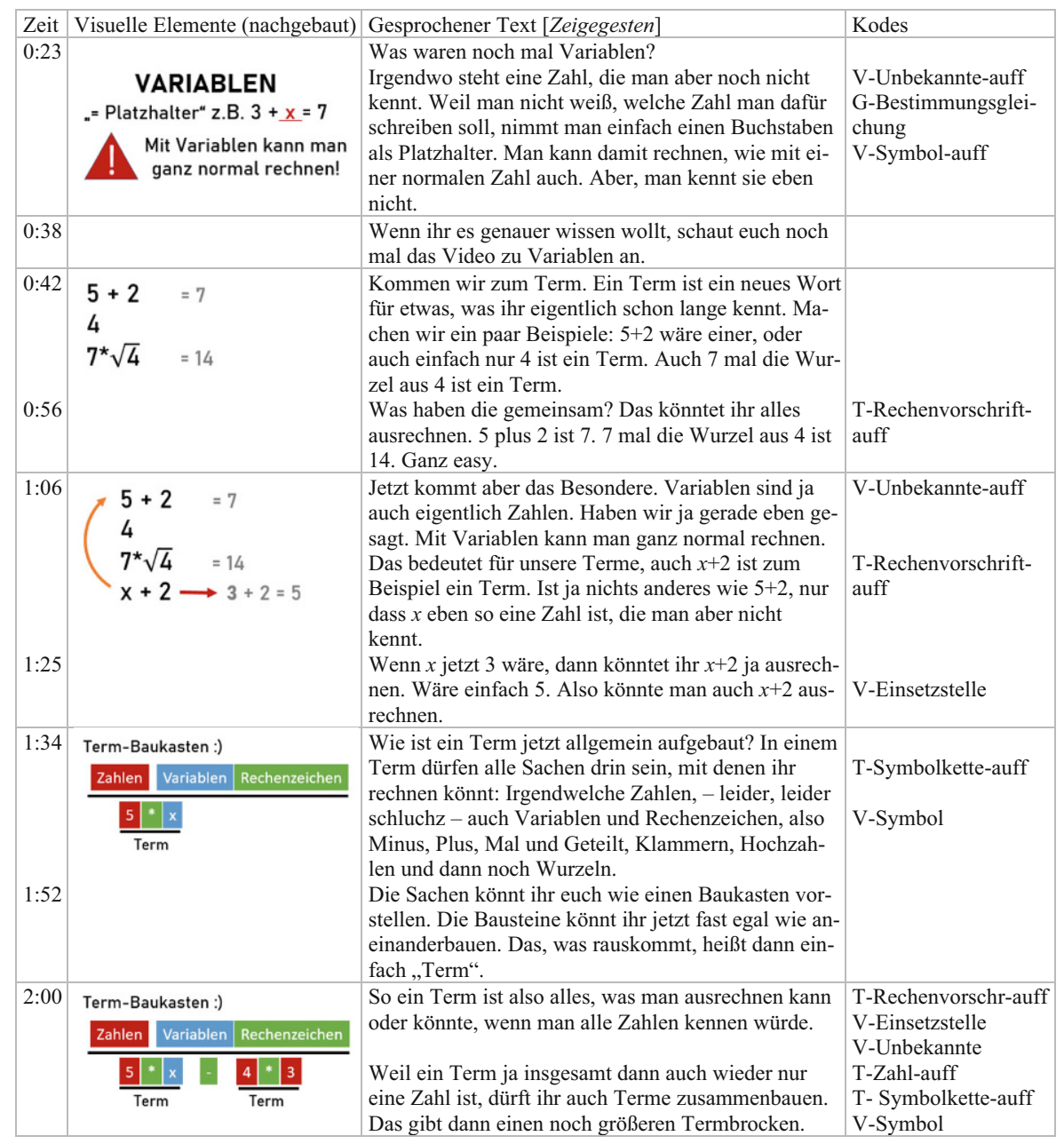

Abb. 1 Transkriptausschnitt zu Erklärvideo V2 (Simpleclub)

V2 des Kanals Simpleclub (Abb. 1) zeigt, dass YouTube-Erklärvideos durchaus umfassend die Deutungen des Variablen-, Term- und Gleichungskonzepts adressieren können.

Analyse des Vorkommens: Variable und Term werden im Videoausschnitt in Abb. 1 in Einsetz- und in Unbekannten-Deutung thematisiert, während das Verstehenselement der Bestimmungsgleichung visuell aufgerufen wird. Neben der Vielfalt der vorkommenden Verstehenselemente zeigt der Transkriptausschnitt auch, dass Verstehenselemente in unterschiedlicher Qualität und Tiefe vorkommen: Während die Bestimmungsgleichung überhaupt nicht sprachlich expliziert wird, wird die Variable sprachlich fokussiert als ,Zahl, die man aber noch nicht kennt“ (V2, 0:23), und im Rahmen einer kurzen Wiederholung aufgefaltet. Umfassend wird der Term als 
Rechenvorschrift aufgefaltet: Ausgehend von einer Verankerung in numerischen Beispielen (V2, 0:56) erfolgt eine Algebraisierung der Vorstellung der Rechenvorschrift durch den Einbezug unbekannter Zahlen (V2, 1:06), die in einem zusammenfassenden Merksatz verdichtet wird: ,ein Term ist also alles, was man ausrechnen kann oder könnte“ (V2, 2:00). Im Rahmen dieser umfassenden Auffaltung des Terms als Rechenvorschrift wird das Verstehenselement der Variable als Einsetzstelle zwar genutzt (V2, 1:25) bzw. dessen Verständnis vorausgesetzt (V2, 2:00), aber selbst nicht explizit erklärt (etwa als „In Variablen lassen sich verschiedene Zahlen einsetzen“).

Analyse des Auffaltens: Anknüpfend an Wittwer und Renkl (2008) ist davon auszugehen, dass vor allem leistungsschwächere Lernende für eine erfolgreiche Nutzung der Erklärangebote auf eine Auffaltung der Verstehenselemente angewiesen sind. Bspw. erwarten die Videohersteller nicht, dass die implizit adressierte Bestimmungsgleichung von Lernenden mit diesem Video neu mental konstruierbar ist, dazu verweisen sie (V2, 0:38) auf das Vorgängervideo (V1). Die Verstehensangebote der Erklärvideos müssen daher auch über das Auffalten der vorkommenden Verstehenselemente erfasst werden.

Tab. 5 zeigt im oberen Teil die Anzahl der 50 Erklärvideos, in denen die Deutungen der Konzepte Variable, Term und Gleichung vorkommen bzw. aufgefaltet werden, sowie die Anzahl der Videos, in denen keine Deutungen vorkommen bzw. aufgefaltet werden.

Alle relevanten Deutungen kommen in den untersuchten Erklärvideos vor und werden auch aufgefaltet. Allerdings zeigt sich für alle Verstehenselemente ein markanter Unterschied in den Häufigkeiten des bloßen Adressierens der relevanten Verstehenselemente und dem für den Verständnisaufbau bedeutsamen Auffalten derselben: Keine der Deutungen wird in mehr als $50 \%$ der Videos, in denen sie vorkommt, auch aufgefaltet. Für den Term dominiert die Einsetz-Deutung, während Gleichungen schwerpunktmäßig in der Unbekannten-Deutung angesprochen und aufgefaltet werden. Die Variable wird etwa gleichhäufig als Unbekannte (25/12) und Unbestimmte (2518) gedeutet. Insgesamt sind Vorkommen und Auffalten der EinsetzDeutungen der drei Konzepte (33|23) ebenso häufig wie die Unbestimmte und Unbekannte zusammen (Videos, in denen mindestens eines von beiden vorkommt/ aufgefaltet wird: 38124). Die Häufigkeiten des Vorkommens und Auffaltens spiegeln demnach die didaktische Relevanz der Verstehenselemente nicht angemessen wider, insbesondere nicht für die Unbestimmte.

Der mittlere Teil der Tab. 5 zeigt die gleichen Zahlen für die Fokusvideos, bspw. fokussiert das obige Erklärvideo von Simpleclub (Abb. 1) auf Terme und Gleichungen und setzt das Variablenkonzept voraus (vgl. Tab. 3). Wie zu erwarten, kommen in vielen Fokusvideos Deutungen der fokussierten Konzepte tatsächlich vor und werden aufgefaltet. In lediglich zwei Term- und einem Gleichungsvideo kommen gar keine Deutungen vor, hier wird hinsichtlich Verstehensorientierung nicht über das Relevante der Konzepte gesprochen. In 18 Fokusvideos für Terme falten 7 (d. h. $39 \%$ ) keine Deutung für Terme auf, sondern verbleiben im Symbolischen. Auch die Einschränkung auf den Fokus der Videos ist also kein Garant für das didaktisch bedeutsame Auffalten der Verstehenselemente. 
Tab. 5 Vorkommen und Auffalten der Variablen-Deutungen in drei algebraischen Konzepten in allen 50 YouTube-Erklärvideos (oben), den jeweiligen Fokusvideos (Mitte), den Kalkülvideos (unten)

\begin{tabular}{|c|c|c|c|c|c|c|c|c|c|}
\hline & \multirow[b]{2}{*}{$\begin{array}{l}\text { Anzahl } \\
\text { Videos }\end{array}$} & \multicolumn{2}{|c|}{ Einsetz-Deutung } & \multicolumn{2}{|c|}{$\begin{array}{l}\text { Unbekannten- } \\
\text { Deutung }\end{array}$} & \multicolumn{2}{|c|}{$\begin{array}{l}\text { Unbestimmten- } \\
\text { Deutung }\end{array}$} & \multicolumn{2}{|c|}{ Keine Deutung } \\
\hline & & $\begin{array}{l}\text { Vorkom- } \\
\text { men }\end{array}$ & $\begin{array}{l}\text { Auffal- } \\
\text { ten }\end{array}$ & $\begin{array}{l}\text { Vorkom- } \\
\text { men }\end{array}$ & $\begin{array}{l}\text { Auffal- } \\
\text { ten }\end{array}$ & $\begin{array}{l}\text { Vorkom- } \\
\text { men }\end{array}$ & $\begin{array}{l}\text { Auffal- } \\
\text { ten }\end{array}$ & $\begin{array}{l}\text { Vorkom- } \\
\text { men }\end{array}$ & $\begin{array}{l}\text { Auffal- } \\
\text { ten }\end{array}$ \\
\hline \multicolumn{10}{|c|}{ Alle 50 Videos } \\
\hline Variable & 50 & 32 & 14 & 25 & 12 & 25 & 8 & 8 & 27 \\
\hline Term & 50 & 32 & 15 & 3 & 1 & 17 & 7 & 16 & 35 \\
\hline Gleichung & 50 & 13 & 6 & 19 & 9 & 10 & 1 & 21 & 37 \\
\hline Gesamt & 50 & 33 & 23 & 26 & 15 & 26 & 10 & 8 & 20 \\
\hline \multicolumn{10}{|c|}{ Fokusvideos } \\
\hline Variable & 13 & 11 & 9 & 10 & 6 & 11 & 6 & 0 & 1 \\
\hline Term & 18 & 16 & 11 & 2 & 1 & 11 & 7 & 2 & 7 \\
\hline Gleichung & 8 & 6 & 5 & 6 & 4 & 1 & 0 & 1 & 2 \\
\hline \multicolumn{10}{|c|}{ Kalkülvideos } \\
\hline- & 5 & 2 & 1 & 0 & 0 & 1 & 0 & 3 & 4 \\
\hline
\end{tabular}

Der untere Teil von Tab. 5 zeigt, dass auch in den Kalkülvideos, die einen prozeduralen Fokus intendieren, Verstehenselemente vorkommen und aufgefaltet werden können, auch wenn dies erwartungsgemäß nicht die Regel ist.

Sprachliche Realisierungen des Auffaltens: In jedem der 13 Fokusvideos zu Variablen werden entweder Unbestimmte oder Unbekannte angesprochen, davon in 11 (85\%) auch aufgefaltet. Die genaueren Analysen zeigen (nicht in Tab. 5), dass zwar in 8 dieser 13 Fokusvideos sowohl Unbekannte als auch Unbestimmte vorkommen, aber nur in einem dieser Videos beide aufgefaltet werden. Tab. 6 zeigt typische sprachliche Realisierungen der Auffaltungen der Unbekannten- und UnbestimmtenDeutungen der Variable in den Fokusvideos. Die Unbekannte wird in der sinnstiftenden Tätigkeit des Findens der Unbekannten ,,aus der 1. Klasse“ (V17, 0:40) rein innermathematisch thematisiert, die Unbestimmte in reichhaltigen Sachkontexten (Streichholzmuster, Eintrittspreise) in Verallgemeinerungstätigkeiten entwickelt, um leichter „öfter mal das Gleiche“(V37, 5:15) zu rechnen.

Inhaltlich und sprachlich sind insbesondere die Auffaltungen der Unbestimmten von einer großen Bandbreite gekennzeichnet (Tab. 6). Während es in den Fokusvideos mit Schwerpunkt auf die Unbekannte selbstverständlich ist, dass die Suche nach unbekannten Zahlen als sinnstiftende Tätigkeit expliziert wird, wird der Zweck der kognitiven Entlastung durch das Beschreiben allgemeiner Zusammenhänge für die Unbestimmte nur in einem Video explizit aufgefaltet (V37, 5:15). Sprachlich zeigen sich Unterschiede im Allgemeinheitsgrad der Auffaltung der Unbestimmten: Lediglich 2 Erklärvideos (V23, V37) erläutern die Unbestimmte allgemein-abstrakt, während die übrigen 4 nur beispielgebundene Auffaltungen im Sachkontext anbieten. Abstraktion und sprachliche Verallgemeinerung werden hier also den Zuschauenden selbst überlassen.

Zusammenfassend lassen sich die Forschungsfragen FF1a und FF1b wie folgt beantworten: Die relevanten Deutungen der Konzepte Variable, Term und Gleichung 
Tab. 6 Typische Auffaltungen der Unbekannten und Unbestimmten aus Fokusvideos der Variable

\section{Auffaltung der Unbekannten:}

- Auffaltung mit Explikation der sinnstiftenden Tätigkeit: „Ich zeig dir mal eine Aufgabe aus der 1. Klasse. 4 plus Kästchen ist gleich 7. Das Kästchen stand für eine Zahl, die noch gefunden werden muss. [...] Mathematiker verwenden statt Kästchen für Variablen meistens kleine Buchstaben [...]. Warum machen Mathematiker sowas? Ganz einfach, [...] wenn sie eine noch unbekannte Zahl suchen, so wie bei den Kästchen eben.“(V17, 0:40)

Auffaltung der Unbestimmten:

- Auffaltung mit Explikation der sinnstiftenden Tätigkeit: „Solche Terme [7x-3] werden dann aufgestellt, wenn man öfter mal das Gleiche rechnen muss mit [...] verschiedenen Zahlen [...], das ist quasi wie ein kleines Computerprogramm, was abläuft." (V37, 5:15)

- Allgemein-abstrakte Auffaltung: „Eine Variable ist eigentlich nichts anderes als ein Symbol, dessen Wert variieren kann, daher Variable. Also der Wert des Symbols kann von Mal zu Mal unterschiedlich sein, mal ist der Wert höher, mal ist der Wert niedriger." (V23, 4:58)

- Beispielgebundene innermathematische Auffaltung: „Wir sollen hier jede Zahl, die wir für x einsetzen, mit 7 multiplizieren und danach noch 3 abziehen." (V37, 5:25)

- Beispielgebundene Auffaltung im Sachkontext: „Pro Person würde man $17 €$ bezahlen, also $17 €$ mal die Personenanzahl. Und die halte ich jetzt einfach mal mit $x$ hier [fest], das ist unsere Variable. Und es kommen immer, egal wie viele Personen dabei sind, noch [...] einmalige Kosten dazu [...].“ (V21, 3:23)

- Beispielgebundene Auffaltung im Sachkontext mit Aufzählung: „Was für einen Term kann ich denn hier nehmen, um rauszukriegen, wie viel Streichhölzer ich brauche für eine beliebige Größe [von Streichholzquadraten], [...] also x gleich 17, oder $x$ gleich 1211, oder $x$ gleich 7."(V45, 9:44)

Vermischung der Auffaltung von Unbekannter (kursiv) und Unbestimmter (fett):

- Und dieses $x$ [...] [in der Gleichung 3+x=5] nennt man in der Mathematik Variable. [...] Und ja, wie der Name schon sagt, ein variabler Wert kann da hineingesteckt werden oder wir können auch sagen beliebiger Wert oder irgendein Wert. Merkt euch: $x$ ist irgendeine Zahl, die wir herausbekommen sollen. (V20, 3:26)

kommen in den analysierten YouTube-Erklärvideos vor. Sie werden allerdings in höchstens der Hälfte der Videos, in denen sie vorkommen, auch aufgefaltet; einige wesentlich seltener (insbesondere Unbestimmten-Deutung der Variable und Gleichung). Auch in den Fokusvideos der Konzepte Variable, Term und Gleichung ist das Vorkommen und Auffalten der Deutungen nicht garantiert. Für den Term wurde die Einsetz-Deutung als Rechenvorschrift als dominant identifiziert, für die Gleichung die Unbekannten-Deutung als Bestimmungsgleichung. Die Variable wird in den Fokusvideos als Unbekannte und Unbestimmte gedeutet. Vor allem hinsichtlich der Unbestimmten zeigen sich unterschiedliche Allgemeinheitsgrade der sprachlichen Realisierungen der Auffaltungen: von beispielgebundenen Auffaltungen im Sachkontext bis hin zu allgemein-abstrakten Auffaltungen mit Explikation der sinnstiftenden Tätigkeit.

\subsubsection{Vernetzung der Verstehenselemente von Variable, Term und Gleichung}

Die sprachliche explizite Vernetzung von Informationen ist zentral für ein tiefgreifendes Verständnis von Lernenden. Für die zuvor beschriebenen Auffaltungen der Unbestimmten und Unbekannten in den Fokusvideos zur Variable ist diesbezüglich auffällig, dass die sinnstiftenden Tätigkeiten (Unbekannte finden, Zusammenhänge verallgemeinern) sowie die beiden Variablen-Deutungen in keinem Video zueinander in Beziehung gesetzt werden - auch nicht in Video V30, das beide Deutungen 
auffaltet. Stattdessen finden sich sogar immer wieder Passagen in den Videos, in denen die Deutungen der Unbestimmten und der Unbekannten mindestens auf Ebene der verwendeten Sprachmittel vermischt werden (Tab. 6). Die saubere (sprachliche) Abgrenzung der beiden Zahl-Deutungen der Variable ist also ebenso eine didaktisch nicht bewältigte Herausforderung der Fokusvideos wie das explizite Herstellen einer Beziehung zwischen diesen Deutungen und ihren sinnstiftenden Tätigkeiten.

Die sprachlich explizite Vernetzung von Deutungen lässt sich anhand des Erklärvideos von Simpleclub (Abb. 1) für das Termkonzept illustrieren: Terme sind Rechenvorschriften mit unbekannten Zahlen und weil Terme selbst nur (unbekannte) Zahlen sind, dürfen sie zu ,größeren Termbrocken“"verbaut werden (V2, 2:00). Die Einsetz- und Unbekannten-Deutung des Terms werden also vernetzt, indem Beziehungen zwischen den verschiedenen Deutungen des gleichen Konzepts sprachlich explizit erklärt werden. Um verschiedene Deutungen explizit in Beziehung zu setzen, müssen sie selbst hinreichend aufgefaltet werden, weshalb das Auffalten von Verstehenselementen als notwendige Bedingung für Vernetzung betrachtet wird.

Insgesamt falten 15 verschiedene Videos mindestens zwei verschiedene Verstehenselemente eines der Konzepte Variable, Term oder Gleichung auf, nur 10 dieser Videos vernetzen die aufgefalteten Verstehenselemente auch sprachlich explizit: 7 dieser Videos vernetzen Deutungen des Variablenkonzepts, 5 des Term- und 4 des Gleichungskonzepts. Abb. 2 zeigt, welche Verstehenselemente in wie vielen dieser Videos sprachlich explizit vernetzt werden. Das Herausarbeiten der Beziehung zwischen Unbestimmten- und Unbekannten-Deutungen ist im Videokorpus auch für Term und Gleichung eine nicht bewältigte didaktische Herausforderung.

Das Erklärvideo von Simpleclub (in Abb. 1) erinnert zur Erläuterung des Terms als Rechenvorschrift mit unbekannten Zahlen (V2, 1:06) an die Wiederholung des Variablenkonzepts zuvor (V2, 0:23) und vernetzt so die Deutung der Variable als Unbekannte und des Terms als Rechenvorschrift. Voraussetzung für eine solche Konzept-Vernetzung ist wiederum, dass beide Verstehenselemente aufgefaltet werden. Andernfalls obliegt es den Zuschauenden ihre bereits vorhandenen KonzeptDeutungen zu aktivieren - im Simpleclub-Video etwa dann, wenn die Unbekannte im Kontext einer Bestimmungsgleichung erläutert wird, die Gleichung aber nicht sprachlich thematisiert wird (V2, 0:23).

Zwar kommen in 41 Videos Deutungen verschiedener Konzepte (nur Variable, Term, Gleichung) gemeinsam vor, allerdings werden nur in 18 Videos die Deutungen unterschiedlicher Konzepte auch aufgefaltet. Diese 18 Videos vernetzen dann auch mindestens 2 aufgefaltete Deutungen der verschiedenen Konzepte. Abb. 3 zeigt
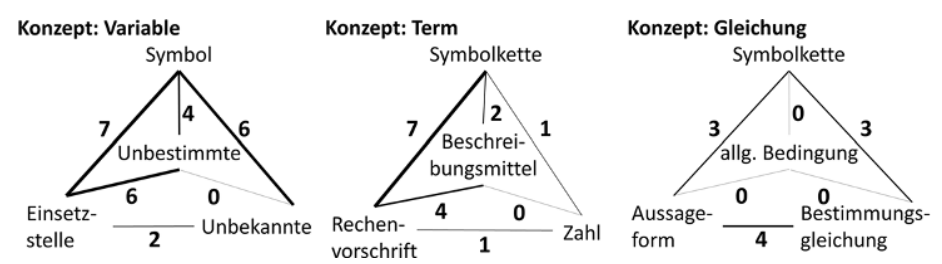

Abb. 2 Sprachlich explizite Vernetzungen zwischen Wissenselementen von drei algebraischen Konzepten in 50 Erklärvideos 
Sprachlich explizite Konzept-Vernetzung

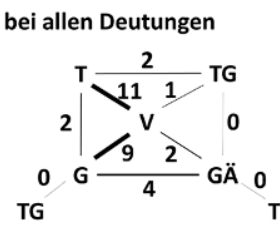

in jeweils verschiedenen Deutungen
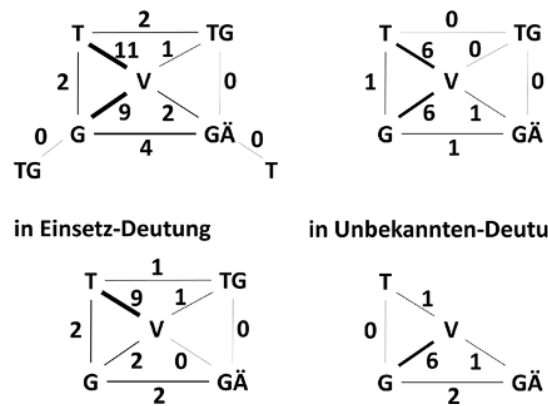

in Unbekannten-Deutung

in Unbestimmten-Deutung
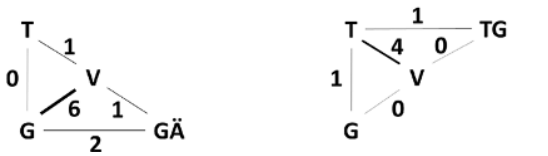

Abb. 3 Konzept-Vernetzung von fünf algebraischen Konzepten in allen bzw. einzelnen Deutungen in 50 Erklärvideos ( $V$ Variable, $T$ Term, $G$ Gleichung, $T G$ Termgleichwertigkeit, $G \ddot{A}$ Gleichungsäquivalenz)

oben links, welche Konzepte in wie vielen Videos vernetzt werden. Bspw. zählt das Simpleclub-Video, in dem der Term als Rechenvorschrift und die Variable als Unbekannte vernetzt wird, zu den 11 Videos, die Variablen- und Term-Deutungen vernetzen.

Differenziert man die Vernetzungen nach den einzelnen Deutungen, so zeigen die übrigen vier Graphen in Abb. 3, wie wenig Deutungen über verschiedene Konzepte hinweg vernetzt werden. In diesen Graphen taucht das Simpleclub-Video (Abb. 1) unter vernetzt ,,in jeweils verschiedenen Deutungen“ auf, da hier der Term in der Einsetz-Deutung und die Variable als Unbekannte vernetzt wird. Die Konzept-Vernetzungen in den erwartbaren (weil inhaltlich eng zusammenhängenden) Deutungen sind zwar am häufigsten, dennoch aber selten: Variable und Gleichung werden in Unbekannten-Deutung in 6 Videos vernetzt, Variable als Einsetzstelle und Term als Rechenvorschrift in 9 Videos.

Zusammenfassend kann Forschungsfrage FF1c also wie folgt beantwortet werden: Konzept- und Deutungs-Vernetzungen verschiedener Deutungen der Konzepte Variable, Term und Gleichung sind möglich, aber selten. Nur 10 der analysierten 50 Erklärvideos vernetzen überhaupt Deutungen innerhalb eines Konzepts und 18 Videos die Deutungen verschiedener Konzepte. Hierbei zeigt sich für die Deutungs-Vernetzung insbesondere, dass Unbekannte und Unbestimmte für alle drei Konzepte in allen 50 Videos beziehungslos nebeneinander stehen bleiben und sie für die Variable auf sprachlicher Ebene sogar vermischt werden. Hinsichtlich der Konzept-Vernetzung werden vor allem eng verwandte Deutungen über verschiedene Konzepte vernetzt.

\subsection{Vorkommen und Auffalten der Kalkülelemente zu Variable, Term, Termgleichwertigkeit, Gleichung und Gleichungsäquivalenz und ihre Vernetzung zu Verstehenselementen}

In Abschn. 2.3 wurde herausgearbeitet, dass die kalkülhafte Nutzung der symbolischen Darstellung von Variable, Term und Gleichung bedeutsam ist, da sie kognitive Entlastung schafft und dadurch den Weg für komplexe kognitive Operationen 
freimacht (Kieran 2013; Hefendehl-Hebeker 2001). Allerdings ist ein verständiger Umgang mit dem Kalkül und den symbolischen Darstellungen auf eine solide Verankerung der Kalkülelemente in den Verstehenselementen der Konzepte angewiesen (Prediger 2009). Daher werden nun das Vorkommen und Auffalten der Kalkülelemente sowie ihre Vernetzung mit relevanten Verstehenselementen untersucht.

\subsubsection{Vorkommen und Auffalten der Kalkülelemente}

Im Erklärvideo von Simpleclub (Abb. 1) wird der Term als Symbolkette aufgefaltet, die bestimmte „Bausteine“ enthalten darf (V2, 1:34). Dabei wird auch die Variable als möglicher Baustein genutzt, ohne aufzufalten, dass Symbole die Variablen darstellen. Tab. 7 stellt eine Erweiterung von Tab. 5 dar, die nicht nur die Verstehens-, sondern auch die Kalkülelemente betrachtet sowie die Wissenselemente zu Termgleichwertigkeit und Gleichungsäquivalenz. Dabei wird wiederum zwischen der Auflistung für alle 50 Videos (oben), den jeweiligen Fokusvideos (Mitte) und den Kalkülvideos (unten) differenziert.

Tab. 7 oben zeigt, dass Kalkülelemente in 49 der 50 Erklärvideos vorkommen und in 45 mindestens eines auch aufgefaltet wird. Sie kommen also häufiger vor als jedes Verstehenselement und werden mit wenigen Ausnahmen (Variable als Einsetzstelle und Unbekannte, Gleichung als Bestimmungsgleichung) auch häufiger aufgefaltet. Besonders deutlich ist dieser Kontrast für die Termgleichwertigkeit und Gleichungsäquivalenz: Aufgefaltet werden $63 \%$ der vorkommenden Kalkülelemente (38|24 bzw. 19|12). D.h. tauchen Umformungen auf, werden ihre Regeln meist ausführlich erläutert. Die zugehörigen Verstehenselemente kommen jedoch selten vor und werden noch seltener aufgefaltet, selbst die am häufigsten vorkommende Gleichungsäquivalenz in Unbekannten-Deutung wird nur viermal aufgefaltet (7/4).

Die Dominanz der Kalkülelemente gilt auch für die Fokusvideos, wie Tab. 7 (Mitte) zeigt. In den Fokusvideos zum Variablen-, Term- und Gleichungskonzept sind die Häufigkeiten der Kalkülelemente denen der Einsetz-Deutung ähnlich, es ist also insgesamt ein ausgewogeneres Verhältnis des Vorkommens von Kalkül- und Verstehenselementen zu verzeichnen.

Die große Präsenz der Kalkülelemente in den Erklärvideos ist durchaus erwartbar, denn das Erklären elementarer Algebra erfordert stets auch das Sprechen über die Struktur von Symbolketten, was u. a. auch das obige Simpleclub-Erklärvideo (Abb. 1) verdeutlicht (V2, 1:34). Allerdings falten 17 der 50 Videos ausschließlich Kalkülelemente auf. 10 der 20 Fokusvideos zur Termgleichwertigkeit und 7 der 18 Fokusvideos zur Gleichungsäquivalenz (Tab. 7 Mitte rechts in Klammern zeigt die Verteilung auf die Fokusvideos; 4 der 21 Videos haben mehr als einen Fokus). Von den 17 rein kalkül-auffaltenden Videos sind nur 4 der Intention nach Kalkülvideos (Tab. 7 unten rechts), d.h. 13 Videos werden den fachdidaktischen Ansprüchen an ihre konzeptuelle Absicht nicht gerecht.

Ein repräsentatives Beispiel für ein solches rein kalkül-auffaltendes Erklärvideo zeigt Abb. 4. Hier wird explizit die Termumformungsregel formuliert und statt Deutungen derselbe „Nachname“ adressiert (V46, 21:03). Die Regel wird in mehreren Beispielen aufgefaltet (V46, 1:07 ff.). Eine Auffaltung der Regel hin zu anderen Ver- 
Tab. 7 Vorkommen und Auffalten der Wissenselemente zu allen fünf Konzepten in allen 50 YouTubeErklärvideos (oben), den jeweiligen Fokusvideos (Mitte), den Kalkülvideos (unten)

\begin{tabular}{|c|c|c|c|c|c|c|c|c|c|}
\hline & \multirow[b]{2}{*}{$\begin{array}{l}\text { Anzahl } \\
\text { Videos }\end{array}$} & \multicolumn{2}{|c|}{$\begin{array}{l}\text { Einsetz-Deu- } \\
\text { tung }\end{array}$} & \multicolumn{2}{|c|}{$\begin{array}{l}\text { Unbekannten- } \\
\text { Deutung }\end{array}$} & \multicolumn{2}{|c|}{$\begin{array}{l}\text { Unbestimmten- } \\
\text { Deutung }\end{array}$} & \multicolumn{2}{|c|}{$\begin{array}{l}\text { Symbol ohne } \\
\text { Deutung }\end{array}$} \\
\hline & & $\begin{array}{l}\text { Vorkom- } \\
\text { men }\end{array}$ & $\begin{array}{l}\text { Auffal- } \\
\text { ten }\end{array}$ & $\begin{array}{l}\text { Vorkom- } \\
\text { men }\end{array}$ & $\begin{array}{l}\text { Auffal- } \\
\text { ten }\end{array}$ & $\begin{array}{l}\text { Vorkom- } \\
\text { men }\end{array}$ & $\begin{array}{l}\text { Auffal- } \\
\text { ten }\end{array}$ & $\begin{array}{l}\text { Vorkom- } \\
\text { men }\end{array}$ & $\begin{array}{l}\text { Auffal- } \\
\text { ten }\end{array}$ \\
\hline \multicolumn{10}{|l|}{ Alle Videos } \\
\hline Variable & 50 & 32 & 14 & 25 & 12 & 25 & 8 & 49 & 12 \\
\hline Term & 50 & 32 & 15 & 3 & 1 & 17 & 7 & 46 & 16 \\
\hline $\begin{array}{l}\text { Termgleich- } \\
\text { wertigkeit }\end{array}$ & 50 & 3 & 2 & - & - & 2 & 1 & 38 & 24 \\
\hline Gleichung & 50 & 13 & 6 & 19 & 9 & 10 & 1 & 24 & 7 \\
\hline $\begin{array}{l}\text { Gleichungs- } \\
\text { äquivalenz }\end{array}$ & 50 & 4 & 3 & 7 & 4 & - & - & 19 & 12 \\
\hline Gesamt & 50 & 33 & 25 & 26 & 15 & 26 & 10 & 49 & 45 \\
\hline \multicolumn{10}{|l|}{ Fokusvideos } \\
\hline Variable & 13 & 11 & 9 & 10 & 6 & 11 & 6 & 13 & $8(1)$ \\
\hline Term & 18 & 16 & 11 & 2 & 1 & 11 & 7 & 17 & $14(2)$ \\
\hline $\begin{array}{l}\text { Termgleich- } \\
\text { wertigkeit }\end{array}$ & 20 & 3 & 1 & - & - & 2 & 1 & 20 & $18(10)$ \\
\hline Gleichung & 8 & 6 & 5 & 6 & 4 & 1 & 0 & 8 & $5(1)$ \\
\hline $\begin{array}{l}\text { Gleichungs- } \\
\text { äquivalenz }\end{array}$ & 18 & 3 & 3 & 7 & 4 & - & - & 17 & $12(7)$ \\
\hline \multicolumn{10}{|l|}{ Kalkülvideos } \\
\hline- & 5 & 2 & 1 & 0 & 0 & 1 & 0 & 5 & $4(4)$ \\
\hline
\end{tabular}

In Klammern Anzahl der Fokus- und Kalkülvideos, in denen nur Kalkülelemente aufgefaltet werden

stehenselementen der Termgleichwertigkeit (dass die Terme beim Einsetzen immer denselben Wert haben oder dasselbe beschreiben) erfolgt im gesamten Video nicht.

Auffällig ist, dass in 9 der 17 rein kalkül-auffaltenden Videos die Fehlvorstellung des Buchstaben als Gegenstand (Küchemann 1981) vorkommt. Insgesamt lässt sich diese Fehlvorstellung in 13 der 50 Videos finden - insbesondere in 10 der 20 Fokusvideos zur Termgleichwertigkeit, in denen sie genutzt wird, um die Umformungsregel des Zusammenfassens zu erklären, wie in Abb. 5 veranschaulicht. Auffällig ist, dass die Erklärung über die Fehlvorstellung häufig als besonders verständlich betont wird, wie etwa der Transkriptausschnitt in Abb. 5 zeigt (V38, 0:16). In $53 \%$ der kalkülorientierten Videos (9 von 17) liegt also eine unpassend realisierte Orientierung am Verstehen der Lernenden vor: eine fachdidaktisch lautere Absicht, die mit fachdidaktisch nicht tragfähigen Mitteln realisiert wird.

Bezüglich der Forschungsfragen FF2a und FF2b lässt sich also festhalten: Die Kalkülelemente sind in fast allen Erklärvideos präsent und werden häufiger aufgefaltet als die inhaltlichen Deutungen. 17 Erklärvideos lassen sich als rein kalkülorientierte Nachhilfe (Rott 2020) beschreiben, 4 davon aus fachdidaktisch nachvollziehbarem Grund (Intention reduziert auf Wiederholung von Verfahren). In 13 Videos findet sich durch Rückgriff auf die Obstsalat-Algebra eine unpassend realisierte Orientierung am Verstehen. 


\begin{tabular}{|c|c|c|c|}
\hline Zeit & $\begin{array}{l}\text { Visuelle Elemente } \\
\text { (nachgebaut) }\end{array}$ & Gesprochener Text [Zeigegesten] & Kodes \\
\hline $0: 41$ & a) $\begin{array}{l}7 a b^{2}+3 a b-4 a^{2} b+5 a b^{2} \\
-2 a b+3 a^{2} b+5 a b-12 a b^{2}\end{array}$ & $\begin{array}{l}\text { Wir fassen hier bei der Aufgabe a) einfach nur die } \\
\text { Zahlen zusammen. [...] Ich sag zu den Buchstaben- } \\
\text { folgen immer Nachnamen [...] Es können nur Zahlen } \\
\text { zusammengefasst hab- werden, die denselben Nach- } \\
\text { namen haben. }\end{array}$ & $\begin{array}{l}\text { TG-Umformung-auff } \\
\text { T-Symbolkette } \\
\text { V-Symbol }\end{array}$ \\
\hline 1:07 & a) $\begin{array}{l}7 a b^{2}+3 a b-4 a^{2} b+\frac{5 a b^{2}}{2 a b}+3 a^{2} b+5 a b-12 a b^{2} \\
-2 a b\end{array}$ & $\begin{array}{l}\text { Der erste Nachname, den wir uns angucken wollen, } \\
\text { ist der Nachname } a b^{2} \text {. Das ist das hier [unterstreicht } \\
\left.7 a b^{2}\right] \text {. Haben wir irgendwo sonst noch Zahlen, die } \\
\text { denselben Nachnamen haben? [...] Das haben wir } \\
\text { hier bei } 5 a b^{2}[\text { unterstreicht] und das haben wir auch } \\
\text { hier bei }-12 a b^{2}[\text { unterstreicht]. [...] Deswegen [...] }\end{array}$ & $\begin{array}{l}\text { T-Symbolkette } \\
\text { V-Symbol }\end{array}$ \\
\hline
\end{tabular}

Abb. 4 Transkriptausschnitt zu Erklärvideo V46 (Herr Mathe)

\begin{tabular}{|c|c|c|c|}
\hline Zeit & Visuelle Elemente (nachgebaut) & Gesprochener Text [Zeigegesten] & Kodes \\
\hline $0: 16$ & & $\begin{array}{l}\text { Wir fangen }[\ldots] \text { an, einfach mal Terme zusammenzu- } \\
\text { fassen. Wir machen es so, dass jeder es versteht, egal } \\
\text { auf welchem Niveau ihr seid. }[\ldots]\end{array}$ & \\
\hline $\begin{array}{l}2: 16 \\
2: 26\end{array}$ & & $\begin{array}{l}\text { Schauen wir uns jetzt mal ein 3. Beispiel an. }[\ldots] \\
\text { Jetzt können wir natürlich nur Bananen mit Bananen } \\
\text { zusammenrechnen und Äpfel mit Äpfeln. [...] }\end{array}$ & $\begin{array}{l}\text { Buchstabe } \\
\text { AlsGegen- } \\
\text { stand }\end{array}$ \\
\hline 3:00 & $3 b+5 a+4 b+3 a=7 b+8 a$ & $\begin{array}{l}\text { Jetzt können wir wieder Bananen und Äpfel ersetzen } \\
\text { durch Buchstaben. Ganz wichtig ist, dass wir sie aber } \\
\text { mit verschiedenen Buchstaben ersetzen. [...] } \\
\text { Es gilt genau das Gleiche: Wir können nur } b \text { s mit } b \text { s } \\
\text { zusammenfassen. [...] }\end{array}$ & $\begin{array}{l}\text { V-Symbol } \\
\text { T-Symbol- } \\
\text { kette } \\
\text { TG-Umfor- } \\
\text { mung-auff }\end{array}$ \\
\hline
\end{tabular}

Abb. 5 Transkriptausschnitt zu Erklärvideo V38 (LehrerBros)

\subsubsection{Vernetzung von Kalkülementen mit Verstehenselementen}

Rein kalkülorientierte Erklärvideos verpassen die Anbindung der Umformungsregeln und Symbolketten an inhaltliche Deutungen. Im obigen Simpleclub-Video (Abb. 1) wird dagegen die symbolische Struktur des Terms aus seiner Deutung als Rechenvorschrift abgeleitet, indem erläutert wird, dass Terme zu immer größeren „Termbrocken“ zusammengebaut werden können, weil Terme letztlich auch nur (unbekannte) Zahlen seien (V2, 1:34). Kalkül- und Verstehenselemente können also in Erklärvideos vernetzt werden, was tatsächlich auch in 25 der analysierten Videos erfolgt. Wie bereits Abb. 2 zeigte, erfolgt die Rückbindung der Kalkülelemente an Verstehenselemente unterschiedlich häufig für die einzelnen Konzepte: vor allem für Variablen (11 Videos) und Terme (8 Videos), weniger für Gleichungen (4 Videos). Das Simpleclub-Video (Abb. 1) zählt hier zu den 7 Erklärvideos, die den Term als Symbolkette und als Rechenvorschrift vernetzen.

Abb. 6 zeigt analog die Vernetzung von Kalkül mit inhaltlichen Deutungen für Termgleichwertigkeit (1 Video) und Gleichungsäquivalenz (4 verschiedene Videos). Diese geringen Anzahlen sind dadurch bedingt, dass Deutungen der Umformungen im untersuchten Sample insgesamt sehr selten aufgefaltet werden (Tab. 7). Bzgl. der Umformungen wird in den YouTube-Erklärvideos also großflächig die Anbindung an inhaltliche Deutungen vernachlässigt. 
Abb. 6 Sprachlich explizite Vernetzungen zwischen Wissenselementen von zwei algebraischen Konzepten in $50 \mathrm{Er}$ klärvideos

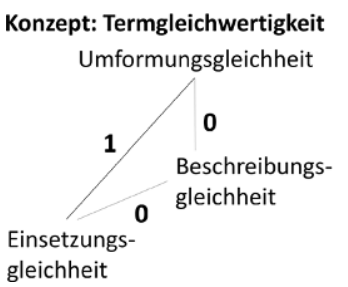

Konzept: Gleichungsäquivalenz

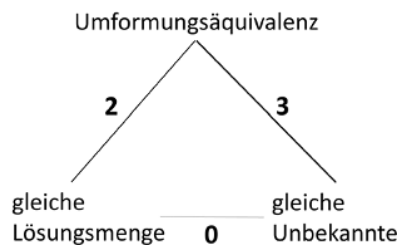

\begin{tabular}{|c|c|c|c|c|c|c|c|}
\hline Zeit & \multicolumn{5}{|c|}{$\begin{array}{l}\text { Visuelle Elemente } \\
\text { (nachgebaut) }\end{array}$} & Gesprochener Text [Zeigegesten] & Kodes \\
\hline $2: 20$ & \multicolumn{5}{|c|}{$\begin{array}{l}T_{1}(x)=x^{2}+1 \\
T_{2}(x)=(x-1) \cdot(x+1) \\
T_{3}(x)=x^{2}-1 \\
G=\{-2 ;-1,5 ; \ldots ; 1\}\end{array}$} & $\begin{array}{l}\text { Da wir diese drei Terme }\left[T_{1}, T_{2}, T_{3}\right] \text { nicht mithilfe der } \\
\text { uns bekannten Rechenregeln umformen können, müs- } \\
\text { sen wir die Äquivalenz auf eine andere Art überprüfen. } \\
\text { Die Idee: Wir erstellen eine numerische Wertetabelle } \\
\text { und vergleichen die Termwerte. [Wertetabelle wird } \\
\text { eingeblendet] Wie du bereits kennen gelernt hast, be- } \\
\text { deutet äquivalent gleichwertig. Wir setzen für } x \text { die } \\
\text { verschiedenen Zahlen der Grundmenge ein und berech- } \\
\text { nen die zugehörigen Termwerte. }\end{array}$ & $\begin{array}{l}\text { TG-Umformung-auff } \\
\text { T-Symbolkette } \\
\text { V-Symbol } \\
\text { TG-Einsetzungs- } \\
\text { gleichheit-auff } \\
\text { T-Rechenvorschrift } \\
\text { V-Einsetzung }\end{array}$ \\
\hline
\end{tabular}

Abb. 7 Transkriptausschnitt zu Erklärvideo V43 (Videolernen)

Eine positive Ausnahme für ein gehaltvolles Zusammenspiel von Inhalt und Kalkül bietet Video V43 (in Abb. 7), in dem Termumformungsregeln wie das Distributivgesetz gesichert werden und der Nachweis der Termgleichwertigkeit von $2(a+4)$ und $2 a+8$ mit Hilfe dieser Regeln ,geübt“ wird. Nun folgen aber nicht, wie in den meisten Termumformungsvideos, weitere vorgerechnete Übungsaufgaben, stattdessen werden momentane Grenzen des Kalküls aufgezeigt und dadurch der Rückgriff auf die Einsetz-Deutung motiviert.

Insgesamt muss Forschungsfrage FF2c also wie folgt beantwortet werden: Unter den Erklärvideos lassen sich solche finden, die gehaltvoll Kalkülelemente mit inhaltlichen Deutungen vernetzen, allerdings sind dies nur für die Variable mehr als $16 \%$ der Videos. Nur 5 Erklärvideos verankern Umformungsregeln für Term und Gleichung in inhaltlichen Deutungen.

\section{Diskussion und Ausblick}

\subsection{Zusammenfassung der Ergebnisse}

Die Analyse der Verstehensangebote von 50 YouTube-Erklärvideos kann die didaktische Einschätzung eines ,,breiten Spektrums“ (Rott 2020) bestätigen und präzisieren: Am einen Ende des Spektrums steht der Befund, dass 17 der 50 Erklärvideos tatsächlich als „kalkülorientierte Nachhilfe“ (Rott 2020) bezeichnet werden müssen (Tab. 7) und 13 Videos eine Fehlvorstellung im Interesse einer ,verständlichen“ Erklärung nutzen (Abschn. 4.2.1). Dieses Ende des Spektrums bestätigt gängige Kritik (Bersch et al. 2020; Klinger und Walter 2020).

Am anderen Ende sind jedoch auch Videos, die Verstehenselemente ansprechen, auffalten und vernetzen; ein Beispiel (Abb. 1) wurde ausführlich diskutiert. Ebenso 
finden sich Erklärvideos, die Kalkülelemente gehaltvoll zu Konzept-Deutungen in Beziehung setzen (Abb. 7). Auch wenn diese eine Ausnahme sind, kann die Ausgangsfrage bejaht werden: Ja, mit Erklärvideos über fachlich anspruchsvolle Konzepte können tatsächlich qualitätsvolle Verstehensangebote erreicht werden! Dies ist für den unterrichtspraktischen Umgang mit Erklärvideos ein weniger pessimistischer Befund als in gängiger Kritik, bestätigt jedoch die hohe Relevanz, für die eigene Lerngruppe eine kluge Wahl zu treffen (Bersch et al. 2020).

Für differenziertere Einblicke wurde in diesem Artikel eine Konzeptualisierung der Qualität von Verstehensangeboten in Erklärvideos über das Vorkommen, Auffalten und Vernetzen relevanter Wissenselemente entwickelt, der auf dem Verstehensmodell von Drollinger-Vetter (2011) und seiner Übertragung auf die Beziehung von Inhalt und Kalkül von Glade und Prediger (2017) beruht. Fokussiert auf den exemplarischen Gegenstand Algebra (Tab. 2) wurden Verstehensangebote gegenstandsbezogen operationalisiert und insofern als genuin fachdidaktischer Kern von Erklärvideos untersucht.

In der vorliegenden Analyse erwies sich die Charakterisierung der Verstehensangebote durch Vorkommen, Auffalten und Vernetzen als besonders gewinnbringend, da hierdurch wichtige Qualitätsunterschiede zwischen den analysierten Erklärvideos erfassbar wurden: Während die relevanten Verstehenselemente zwar in vielen Erklärvideos vorkommen (Einsetz-Deutung in 33 Videos, Unbekannte und Unbestimmte in je 26 Videos), wird kein Verstehenselement in mehr als 50\% der Videos auch aufgefaltet, d.h. explizit erklärt (Tab. 5). Unbekannte und Unbestimmte werden jeweils in 6 der 11 Fokusvideos der Variable aufgefaltet, jedoch nicht zueinander in Beziehung gesetzt (Abschn. 4.1).

Dieser Befund ist für die Algebra-Didaktik auch über Erklärvideos hinaus bedeutsam: Da andere Deutungen in den Videos durchaus auf gelungene Weise miteinander vernetzt werden (etwa Einsetz- und Unbekannten-Deutung im Beispiel aus Abb. 1), liegt in der Vernetzung zwischen Unbestimmter und Unbekannter eine bislang unbewältigte fachdidaktische Herausforderung, deren Prävalenz auch in Schulbüchern zu überprüfen wäre. Dies hängt vermutlich damit zusammen, dass beide Deutungen aus verschiedenen Tätigkeiten ihre Sinnstiftung beziehen (unbekannte Zahlen finden vs. Verallgemeinern). Jedoch werden in vielen Aufgaben, in denen zunächst allgemeine Bedingungen formuliert und dann in Bestimmungsgleichungen uminterpretiert werden, beide Tätigkeiten verknüpft, ohne dass dies für Lernende expliziert wird. Die identifizierten sprachlichen Vermischungen (Tab. 6) sind ein Indikator für diesbezüglich fehlendes didaktisches Problembewusstsein.

Die theoretische Fokussierung der Verstehensangebote auf gegenstandsspezifische Wissenselemente als Verstehens- und Kalkülelemente ermöglicht es zudem, die oberflächliche Unterscheidung zwischen Verstehen und Kalkül aufzulösen und die Anbindung der Kalkülelemente an inhaltliche Verstehenselemente systematisch zu analysieren, auch im Hinblick auf die Auffaltbarkeit und Begründbarkeit der Umformungen (Glade und Prediger 2017). Die Analysen zeigen etwa, dass Kalkülelemente zwar vorkommen (in 49 Videos) und aufgefaltet werden (in 45 Videos, vgl. Tab. 7), allerdings werden sie nur sehr selten in inhaltlichen Deutungen verankert (Abb. 2 und 6). Wir plädieren dringend dafür, die scheinbare Dichotomie zwischen Verstehen und Kalkül durch die vorgeschlagene Operationalisierung zu überwinden. 
Dies ist auch anschlussfähig an den algebradidaktischen Forschungsstand jenseits von Erklärvideos (Kieran 2013).

\subsection{Inhaltliche und methodische Grenzen und Anschlussfragen}

Wie jede Forschung unterliegt auch die hier vorliegende Studie methodischen Einschränkungen. Zunächst ist diesbezüglich festzuhalten, dass nur eines unter mehreren Qualitätsmerkmalen von Erklärvideos analysiert wurde (vgl. Tab. 1): die inhaltliche Qualität der Verstehensangebote.

Marquardt (2016, S. 67 f.) hat bereits darauf hingewiesen, dass die inhaltliche Qualität von Erklärvideos nicht allein durch das Einzelvideo selbst, sondern über komplette Playlists eines Kanals zu einem Thema generiert wird. Daher seien Erklärvideos mit einzelnen Lerneinheiten eines Schulbuchs zu vergleichen, während Playlists Schulbuchkapiteln entsprächen. Marquardts Beobachtung trifft sicherlich auch auf die Verstehensangebote der hier untersuchten Erklärvideos zu und konnte im Simpleclub-Video (Abb. 1) beobachtet werden. Durch die Herstellung von Playlists haben die Kanäle die Möglichkeit, in einzelnen Videos gezielt Themenschwerpunkte zu setzen. Eine Analyse der Verstehensangebote gesamter Playlists könnte also weiteres Vernetzungspotential zu Tage fördern, da gewisse Auffaltungen von Verstehenselementen durch Verweis auf vorangegangene Videos unterlassen werden.

Die Unterrichtsforschungen (Hiebert und Grouws 2007; Drollinger-Vetter 2011) konnten für Mathematikunterricht nachweisen, dass nicht allein die Verstehensangebote für fachlichen Lernerfolg prädiktiv sind, sondern ihre kognitiv aktivierende Verarbeitung durch die einzelnen Lernenden. Folgestudien sollten die Qualität von Verstehensangeboten in Erklärvideos daher auch in ihrem Zusammenspiel mit weiteren Qualitätsmerkmalen (kognitiver Aktivierung, Adaptivität, Tab. 1) und bzgl. lernendenseitiger Verstehensprozesse empirisch untersuchen. Mit Blick auf die kognitive Aktivierung könnten Auslassungen und z. B. Selbsterklärungsprompts interessant sein, die Lernende zu eigenen Auffaltungen und Vernetzungen anregen. Die vorgelegte Konzeptualisierung der Verstehensangebote ermöglicht künftigen lernendenseitigen Nutzungs-Studien die wichtige Unterscheidung zwischen gemeinsamem Auffalten von Verstehenselementen und explizitem sprachlichen Vernetzen.

Die ersten sprachlichen Analysen der Auffaltungen (Tab. 6) geben zudem Hinweise, dass weitere Analysen der sprachlichen Angebote der Videos sowie deren Einfluss auf das Verständnis der Lernenden vielversprechend sind. Zudem sollten die hier nur für 50 Videos zu Variablen, Termen und Gleichungen durchgeführten Analysen auf weitere Videos und andere mathematische Konzepte übertragen werden, denn gerade in den Sprachangeboten könnten relevante weitere Potenziale von Erklärvideos liegen.

Aus unterrichtspraktischer Perspektive ist der Befund sehr heterogener Verstehensangebote ein wichtiger Hinweis auf den Bedarf einer verlässlichen fachdidaktischen Qualitätssicherung, der nicht nur durch Sensibilisierung von Lehrkräften für Qualitätsmerkmale (Marquardt 2016; Klinger und Walter 2020), sondern in künftigen Design-Research-Projekten auch mit eigenen Erklärvideos bearbeitet werden soll. 
Danksagung Wir danken Aylina Westermann, Karin Eckerlin und Maja Motzko für die Mithilfe bei der Auswertung.

Förderung Die Studie gehört zum Projekt MuM-Video - Erklärvideos als Ressource für fach- und sprachintegrierten Mathematikunterricht, das von 2020-24 vom Bundesministerium für Bildung und Forschung gefördert wird (Förderkennzeichen 01JD2001A, Projektleitung S. Prediger und M. Altieri).

Funding Open Access funding enabled and organized by Projekt DEAL.

Open Access Dieser Artikel wird unter der Creative Commons Namensnennung 4.0 International Lizenz veröffentlicht, welche die Nutzung, Vervielfältigung, Bearbeitung, Verbreitung und Wiedergabe in jeglichem Medium und Format erlaubt, sofern Sie den/die ursprünglichen Autor(en) und die Quelle ordnungsgemäß nennen, einen Link zur Creative Commons Lizenz beifügen und angeben, ob Änderungen vorgenommen wurden.

Die in diesem Artikel enthaltenen Bilder und sonstiges Drittmaterial unterliegen ebenfalls der genannten Creative Commons Lizenz, sofern sich aus der Abbildungslegende nichts anderes ergibt. Sofern das betreffende Material nicht unter der genannten Creative Commons Lizenz steht und die betreffende Handlung nicht nach gesetzlichen Vorschriften erlaubt ist, ist für die oben aufgeführten Weiterverwendungen des Materials die Einwilligung des jeweiligen Rechteinhabers einzuholen.

Weitere Details zur Lizenz entnehmen Sie bitte der Lizenzinformation auf http://creativecommons.org/ licenses/by/4.0/deed.de.

\section{Literatur}

Aebli, H. (1994). Denken: Das Ordnen des Tuns. Band II: Denkprozesse. Stuttgart: Klett-Cotta.

Arcavi, A., Drijvers, P., \& Stacey, K. (2017). The learning and teaching of algebra: ideas, insights, and activities. Abingdon: Routledge.

Bednarz, N., Kieran, C., \& Lee, L. (1996). Approaches to algebra: perspectives for research and teaching. In N. Bednarz, C. Kieran \& L. Lee (Hrsg.), Approaches to algebra (S. 3-12). Dordrecht: Kluwer.

Bersch, S., Merkel, A., Oldenburg, R., \& Weckerle, M. (2020). Erklärvideos: Chancen und Risiken. Zwischen fachlicher Korrektheit und didaktischen Zielen. GDM-Mitteilungen, 109, 58-63.

Drollinger-Vetter, B. (2011). Verstehenselemente und strukturelle Klarheit: Fachdidaktische Qualität der Anleitung von mathematischen Verstehensprozessen im Unterricht. Münster: Waxmann.

Findeisen, S., Horn, S., \& Seifried, J. (2019). Lernen durch Videos - Empirische Befunde zur Gestaltung von Erklärvideos. MedienPädagogik, 2019(Occasional Papers), 16-36.

FORSA (2020). Das Deutsche Schulbarometer Spezial Corona-Krise. https://deutsches-schulportal.de/ unterricht/das-deutsche-schulbarometer-spezial-corona-krise/. Zugegriffen: 11. Nov. 2020.

Freudenthal, H. (1973). Mathematics as an educational task. Dordrecht: Reidel.

Glade, M., \& Prediger, S. (2017). Students' individual schematization pathways-empirical reconstructions for the case of part-of-part determination for fractions. Educational Studies in Mathematics, 94(2), 185-203.

Hefendehl-Hebeker, L. (2001). Die Wissensform des Formelwissens. In W. Weiser \& B. Wollring (Hrsg.), Beiträge zur Didaktik der Primarstufe (S. 83-98). Hamburg: Dr. Kovac.

Hiebert, J., \& Carpenter, T.P. (1992). Learning and teaching with understanding. In D. A. Grouws (Hrsg.), Handbook of research on mathematics teaching and learning (S. 65-97). New York: Macmillan.

Hiebert, J., \& Grouws, D. A. (2007). The effects of classroom mathematics teaching on students' learning. In F. K. Lester (Hrsg.), Second handbook of research on mathematics teaching and learning (S. 371-404). Charlotte: Information Age.

vom Hofe, R. (1995). Grundvorstellungen mathematischer Inhalte. Heidelberg: Spektrum.

Höffler, T. N., \& Leutner, D. (2007). Instructional animation versus static pictures: a meta-analysis. Learning and Instruction, 17(6), 722-738.

Kant, J.M., Scheiter, K., \& Oschatz, K. (2017). How to sequence video modeling examples and inquiry tasks to foster scientific reasoning. Learning and Instruction, 52, 46-58.

Kaput, J. J. (2008). What is algebra? What is algebraic reasoning? In J. J. Kaput, D. W. Carraher \& M.L. Blanton (Hrsg.), Algebra in the early grades (S. 5-17). New York: Lawrence Erlbaum. 
Kieran, C. (2004). The core of algebra: reflections on its main activities. In K. Stacey, H. Chick \& M. Kendal (Hrsg.), The future of the teaching and learning of algebra. The 12th ICMI study. New ICMI study series, (Bd. 8, S. 21-33). Dordrecht: Kluwer.

Kieran, C. (2013). The false dichotomy in mathematics education between conceptual understanding and procedural skills: an example from algebra. In K. R. Leatham (Hrsg.), Vital directions for mathematics education research (Bd. 7, S. 153-171). New York: Springer.

Klinger, M., \& Walter, D. (2020). „Ein wahrer Ehrenmann!“ - Wie mathematikhaltige Apps und Videos von Nutzenden bewertet werden. In H.-S. Siller, W. Weigel \& J.F. Wörler (Hrsg.), Beiträge zum Mathematikunterricht 2020 (S. 513-516). Münster: WTM.

Küchemann, D. E. (1981). Algebra. In K. M. Hart (Hrsg.), Children's understanding of mathematics: 11-16 (S. 102-119). London: John Murray.

Kulgemeyer, C. (2018). A framework of effective science explanation videos informed by criteria for instructional explanations. Research in Science Education, 26(1), 1-22.

Kulgemeyer, C., \& Peters, C.H. (2016). Exploring the explaining quality of physics online explanatory videos. European Journal of Physics, 37(6), 1-14.

MacGregor, M., \& Stacey, K. (1997). Students' understanding of algebraic notation: 11-15. Educational Studies in Mathematics, 33(1), 1-19.

Malisani, E., \& Spagnolo, F. (2009). From arithmetical thought to algebraic thought: the role of the "variable". Educational Studies in Mathematics, 71(1), 19-41.

Malle, G. (1993). Didaktische Probleme der elementaren Algebra. Wiesbaden: Vieweg+Teubner.

Marquardt, K. (2016). Beurteilungsraster für Mathematik-Erklärvideos: Chancen, Grenzen und Durchführung mittels Resultaten aus der Schulbuchforschung (Diplomarbeit). tinyurl.com/y87hmhm3. Zugegriffen: 11. Nov. 2020. Universität Wien.

Mayer, R.E. (2009). Multimedia learning. Cambridge: Cambridge University Press.

Mayring, P. (2015). Qualitative Inhaltsanalyse: Grundlagen und Techniken. Weinheim: Beltz.

MPFS - Medienpädagogischer Forschungsverbund Südwest (2020). JIM-Studie 2020: Jugend, Information, Medien. Basisuntersuchung zum Medienumgang 12- bis 19-Jähriger. https://www.mpfs.de/ fileadmin/files/Studien/JIM/2020/JIM-Studie-2020_Web_final.pdf. Zugegriffen: 24. Mai 2021.

Prediger, S. (2009). Inhaltliches Denken vor Kalkül: Ein didaktisches Prinzip zur Vorbeugung und Förderung bei Rechenschwierigkeiten. In A. Fritz \& S. Schmidt (Hrsg.), Fördernder Mathematikunterricht in der Sekundarstufe I (S. 213-234). Weinheim: Beltz.

Prediger, S. (2020). Variable, Terme, Formeln und Gleichungen in Klasse 7-9. In S. Prediger (Hrsg.), Sprachbildender Mathematikunterricht in der Sekundarstufe (S. 162-166). Berlin: Cornelsen.

Prediger, S., \& Krägeloh, N. (2016). "X-arbitrary means any number, but you do not know which one": the epistemic role of languages while constructing meaning for the variable as generalizers. In A. Halai \& P. Clarkson (Hrsg.), Teaching and learning mathematics in multilingual classrooms (S. 89-108). Rotterdam: Sense.

Reusser, K., \& Pauli, C. (2013). Verständnisorientierung in Mathematikstunden erfassen. Ergebnisse eines methodenintegrativen Ansatzes. Zeitschrift für Pädagogik, 59(3), 308-335.

Rott, B. (2020). Videos für die Fernlehre. Von einem der auszog, Videos zu produzieren. GDM-Mitteilungen, 109, 49-50.

Usiskin, Z. (1988). Conceptions of school algebra and uses of variables. In A. F. Coxford (Hrsg.), Yearbook 1988. The ideas of algebra, K-12 (S. 8-19). Reston: National Council of Teachers of Mathematics.

Weeraratne, B., \& Chin, B. (2018). Can Khan Academy e-learning video tutorials improve mathematics achievement in Sri Lanka? International Journal of Education and Development using Information and Communication Technology, 14(3), 93-112.

Wirtz, M.A., \& Caspar, F. (2002). Beurteilerübereinstimmung und Beurteilerreliabilität. Göttingen: Hogrefe.

Wittwer, J., \& Renkl, A. (2008). Why instructional explanations often do not work: a framework for understanding the effectiveness of instructional explanations. Educational Psychologist, 43(1), 49-64.

Wolf, K. D. (2015). Bildungspotenziale von Erklärvideos und Tutorials auf YouTube. Medien + Erziehung, 1(59), 30-36. 